\title{
WILEY-VCH
}

\section{Nanotheranostic Interface Based on Antibiotic-Loaded Conducting Polymer Nanoparticles for Real-Time Monitoring of Bacterial Growth Inhibition}

Hamidreza Enshaei, Anna Puiggalí-Jou, * Luis J. del Valle, Pau Turon, Núria Saperas* and Carlos Alemán*

H. Enshaei, Dr. A. Puiggalí-Jou, Dr. L. J. del Valle, Dr. N. Saperas, Prof. Dr. C. Alemán Departament d'Enginyeria Química, EEBE, Universitat Politècnica de Catalunya, C/ Eduard Maristany 10-14, Ed. I2, 08019 Barcelona, Spain

E-mail: anna.puiggali@upc.edu,nuria.saperas@upc.edu and carlos.aleman@upc.edu $(\mathrm{CV}$

Dr. A. Puiggalí-Jou, Dr. L. J. del Valle, Prof. Dr. C. Alemán

Barcelona Research Center for Multiscale Science and Engineering, EEBE, Universitat Politècnica de Catalunya, C/ Eduard Maristany 10-14, Ed. C, 08019 Barcelona, Spain

Dr. P. Turon

B. Braun Surgical, S.A. Carretera de Terrassa 121, 08191 Rubí (Barcelona), Spain

Dr. C. Alemán

Institute for Bioengineering of Catalonia (IBEC), The Barcelona Institute of Science and Technology, Baldiri Reixac 10-12, 08028 Barcelona, Spain

Keywords: bacterial detection, chloramphenicol, conducting polymers, drug release, electrochemical sensors, electrostimulated release, polythiophene,

Conducting polymers have been increasingly used as biologically interfacing electrodes for biomedical applications due to their excellent and fast electrochemical response, reversible doping-dedoping characteristics, high stability, easy processability and biocompatibility.

These advantageous properties can be used for the rapid detection and eradication of infections associated to bacterial growth since these are a tremendous burden for individual patients as well as the global healthcare system. Herein, we present a smart nanotheranostic electroresponsive platform, which consists of chloramphenicol (CAM)-loaded in poly(3,4ethylendioxythiophene) nanoparticles (PEDOT/CAM NPs) for concurrent release of the antibiotic and real-time monitoring of bacterial growth. PEDOT/CAM NPs, with an antibiotic loading content of $11.9 \pm 1.3 \% \mathrm{w} / \mathrm{w}$, have been proved to inhibit the growth of Escherichia coli and Streptococcus sanguinis due to the antibiotic release by cyclic voltammetry.

Furthermore, in situ monitoring of bacterial activity has been achieved through the electrochemical detection of $\beta$-nicotinamide adenine dinucleotide (NADH), a redox active 
specie produced by the microbial metabolism that diffuse to the extracellular medium. According to these results, the proposed nanotheranostic platform has great potential for realtime monitoring of the response of bacteria to the released antibiotic, contributing to the evolution of the personalized medicine.

\section{Introduction}

Even though microbial pathogens have always accompanied humanity, they still cause a significant morbidity and mortality, affecting over 250 million people worldwide per year. ${ }^{[1]}$ The main sources of infection include water and food contamination, as well as body fluids. In addition, contamination can be easily spread in clinical facilities developing nosocomial infections, where immune-compromised patients are in contact with microbial pathogens residing on hospital. Most alarmingly, common drugs are becoming less effective against well-known infections and new pathogens are continually being found. ${ }^{[2]}$ While much effort and funds have been devoted to the design and development of new antibacterial vaccines and drugs, more attention is needed to limit and control infectious outbreaks. It is necessary to promote the implementation of early detection systems that can warn in qualitative and quantitative manners. Hence, there is an urgent need for an early and rapid diagnostic tool that can detect pathogenic microorganisms and, at the same time, assess their responsiveness to antimicrobial drugs. Electrochemical biosensors for early pathogen detection ${ }^{[3]}$ are a promising alternative to conventional methodologies, which are mainly based on cell culturing, molecular methods and mass spectrometry. ${ }^{[4]}$ Electrochemical methods offer many advantages, the main ones being rapid detection, high sensitivity, low-cost, simplicity, and lack of time-consuming pre-treatments. Other interesting advantages include that most electrochemical-based sensors are label-free devices and can be developed miniaturized, flexible, disposable, wearable and/or implantable formats. ${ }^{[5]}$ Therefore, electrochemical 


\section{WILEY-VCH}

strategies have been recognized as effective tools for the successful detection of whole bacteria, cellular derived signalling molecules, bacterial metabolites and by-products, and enzymes.

Pathogen identification via direct detection of cellular biogenic metabolites is a straightforward electrochemical sensing approach that does not require the use of highly specialized materials and/or instruments. Within this context, voltammetric techniques have been previously used to sense bacterial metabolites and by-products, such as pyoverdine, pyocyanin and 2-heptyl-3-hydroxy-4-quinolone and 2-heptyl-4-hydroxyquinoline.

Nevertheless, these products are just produced by some type of bacteria. For example, Buzid et al. ${ }^{[6]}$ detected pyocyanin, 2-heptyl-3-hydroxy-4-quinolone and 2-heptyl-4hydroxyquinoline from Pseudonomas aeruginosa (P. aeruginosa) cultures using differential pulse voltammetry and employing a thin electrode of boron-doped diamond improved with cationic surfactant hexadecyltrimethylammonium bromide. Sismaet et al. ${ }^{[7]}$ used square-wave voltammetry to measure the concentration of pyocyanin of $P$. aeruginosa isolates from different clinical patients. Results indicated that all isolates were measurable and pyocyanin concentration was correlated with patient symptoms and comorbidity. Sedki et al. ${ }^{[8]}$ utilized reduced graphene oxide-hyperbranched chitosan nanocomposites to monitor Escherichia coli (E. coli) and P. aeruginosa growth by following the oxidation of bacteria metabolites (i.e. undefined electrochemically active secreted biomolecules). However, other authors have been focused on the electrochemical detection of well-defined redox active species released by bacteria to the extracellular environment, such as low molecular weight thiols, ${ }^{[9]} \beta$ nicotinamide adenine dinucleotide $(\mathrm{NADH}),{ }^{[10]} \beta$-nicotinamide adenine dinucleotide phosphate (NADPH) and flavins. ${ }^{[11]}$ On the other hand, antibiotic-loaded biomaterials are under continuous development for infection control. Indeed, antibiotics are routinely delivered in orthopaedic applications such as prosthetic hips. ${ }^{[12]}$ Nevertheless, many times there is no control over the quantity of released drug and, frequently, the amount of antibiotic 


\section{WILEY-VCH}

loaded on the implant is at excessive concentration (i.e. much higher than the minimum inhibitory concentration). Despite of this limitation, some antibiotics, such as ciprofloxacin hydrochloride, ${ }^{[13]}$ gentamicin, ${ }^{[14]}$ ampicillin, ${ }^{[14]}$ and vancomycin, ${ }^{[15]}$ have been loaded on polymeric platforms for subsequent controlled release in response to external stimulation (e.g. electrical ${ }^{[13]}$ and changes in $\mathrm{pH}^{[14,15]}$ ) rather than diffusion or biodegradation of the polymeric matrix. Moreover, the integration of the two functionalities, controlled antibiotic release to kill bacteria using minimal antibiotic concentrations and real-time pathogen monitoring, in a single polymeric device have not been tried yet.

In this work, we present a system capable of slowly release a previously loaded antibiotic and, at the same time, recording the bacterial growth and inhibition. This approach allows us to envisage a future where the infection treatment and testing efficacy of the antibiotic treatment can be performed simultaneously. For this purpose, poly(3,4-ethylenedioxythiophene) (PEDOT) nanoparticles (NPs) loaded with chloramphenicol (CAM) have been synthesized and, subsequently, fixed to a screen-printed carbon electrode (SPCE) using a chitosan layer. CAM is a wide-spectrum antibiotic that inhibits protein synthesis blocking the ribosome functions, ${ }^{[16]}$ while PEDOT is a conducting polymer with superior capacitive performance, high conductivity, stability in aqueous media and biocompatibility. ${ }^{[17]}$ Moreover, PEDOT NPs were successfully used to detect extracellular NADH coming from the respiration reactions of bacteria that diffuses across cell membrane without the interference from the eukaryotic NADH pool, allowing to distinguish prokaryotic from eukaryotic cells. ${ }^{[10]}$ Overall, this work presents an efficient approach for a rapid, real-time and accurate monitoring of the susceptibility of bacteria to the effect of CAM antibiotic. The proposed system is expected to lead to a more personalized medicine using simple materials and equipment that identify more efficient and specific treatments in a short period of time.

\section{Results and discussion}




\section{WILEY-VCH}

\subsection{Chloramphenicol encapsulation}

The conducting polymer used in this work is PEDOT, which is frequently employed for the fabrication of biomedical devices because of its outstanding electrochemical properties, biocompatibility and stability in continuous operation. ${ }^{[17]}$ Furthermore, this conducting polymer was found to be very effective for the electro-stimulated release of encapsulated drugs. ${ }^{[18,19]}$ A representative and common antibiotic, CAM, was loaded into the PEDOT NPs. In this work, unloaded PEDOT NPs (control) and CAM-loaded PEDOT NPs (hereafter PEDOT/CAM NPs) were prepared by in situ emulsion polymerization as shown in Scheme 1 (details are provided in the Supporting Information). The encapsulation of the antibiotic in the NPs is promoted by the attractive interactions between the negatively charged (oxygen) and the electronegative (chlorine) atoms of CAM and the oxidized PEDOT chains (Scheme 1). Moreover, the nitro group in CAM, a strong electron-withdrawing group, reduced the electron density of PEDOT chains, acting as electron acceptor.

The average diameter of the resulting PEDOT and PEDOT/CAM NPs, as measured by dynamic light scattering (DLS), was $208 \mathrm{~nm}$ and $215 \mathrm{~nm}$, respectively (Figure 1a). In both cases, the polydispersity (PDI) values were smaller than 0.3 . As the numerical value of PDI ranges from 0.0 (for an ideal uniform sample with respect to the particle size) to 1.0 (for a highly polydisperse sample with multiple particle size distribution), the obtained values indicate a relatively narrow size distribution. The zeta $(\zeta)$-potential of PEDOT NPs, which was negative (Figure 1a), became $\sim 7 \mathrm{mV}$ less negative when CAM was loaded. This is consistent with the $\zeta$-potential obtained for CAM solutions (Figure S1), which are significantly lower than for PEDOT NPs (i.e. $-7.1 \pm 3.2 \mathrm{mV}$ and $-4.7 \pm 2.6 \mathrm{mV} \mathrm{mV}$ for 0.1 for 0.1 and $0.5 \mathrm{mg} / \mathrm{mL}$ CAM solutions, respectively, against, $-26.0 \pm 7.3 \mathrm{mV}$ for PEDOT NPs). These results indicate that the presence of the drug partially neutralized the overall negative charge coming from the DBSA. 


\section{WILEY-VCH}

The LC (Eq. S1) was determined by dissolving the loaded drug in ethanol and using UV-Vis absorbance for quantification (Figure 1b). The complete procedure is described in the Supporting Information, while Figures S2-S3 display the calibration curve obtained in the different media used in this work. It has been previously described that PEDOT NPs can act as excellent therapeutic molecule carriers and attain successful electronic triggered release. ${ }^{[18,19]}$ However, the encapsulation efficiency for small molecules is challenging, usually leading to low values. In this case, the LC was $11.9 \pm 1.3 \%$, which was expressed as mass of loaded CAM with respect to the total mass. This value is larger than those achieved for other small drugs, such as curcumin (CUR) and piperine (PIP) (i.e. LC=5.9 $\pm 1.6 \mathrm{wt} \%$ and $8.0 \pm 0.4 \mathrm{wt} \%$ for PEDOT/CUR and PEDOT/PIP NPs, respectively) ${ }^{[18]}$ but lower than obtained for negatively charged pentapeptides, hereafter denoted generically PPEP (LC= 33.8 $\pm 4.3 \mathrm{wt} \%$ and $43.2 \pm 2.9 \mathrm{wt} \%$, depending on the PPEP sequence), ${ }^{[19]}$ which acted as dopant anions.

The presence of the drug in PEDOT/CAM NPs was characterized by FTIR and Raman spectroscopy. Figure 1c compares the FTIR spectrum of the free CAM, PEDOT NPs and PEDOT/CAM NPs. As expected, the absorption bands found in the PEDOT NPs spectrum mainly correspond to the characteristic peaks of PEDOT chains, which appear at 1644 and $1477 \mathrm{~cm}^{-1}$ ( $\mathrm{C}=\mathrm{C}$ stretching), $1350 \mathrm{~cm}^{-1}$ (C-C stretching), 1215 and $1058 \mathrm{~cm}^{-1}(\mathrm{C}-\mathrm{O}-\mathrm{C}$ vibrations) and $840 \mathrm{~cm}^{-1}$ (stretch of the $\mathrm{C}-\mathrm{S}$ bond in the thiophene ring). Also, weak but clearly defined bands attributed to residual DBSA molecules were detected at 2922 and 2853 $\mathrm{cm}^{-1}$ (aliphatic $-\mathrm{CH}_{2}$ and $-\mathrm{CH}_{3}$ stretching), and $1644 \mathrm{~cm}^{-1}(\mathrm{C}=\mathrm{C}$ stretching from the phenyl ring). Free CAM exhibits the characteristic absorption peaks at 3333, 3264 and $3073 \mathrm{~cm}^{-1}$, which have been assigned to the stretch of $\mathrm{O}-\mathrm{H}, \mathrm{N}-\mathrm{H}$ and $\mathrm{C}-\mathrm{H}$ bonds, respectively. ${ }^{[20]}$ Also, absorption bands at $1680 / 1563 \mathrm{~cm}^{-1}$ due to amide I/amide II of the 2,2-dichlor-acetamide moiety, and at $1513 / 1340 \mathrm{~cm}^{-1}$ due to nitro/nitro-phenyl group are present. Finally, PEDOT/CAM NPs present mostly the bands associated with PEDOT NPs but also a very 


\section{WILEY-VCH}

weak band associated with CAM presence at $3073 \mathrm{~cm}^{-1}$, which corresponds to the $\mathrm{C}-\mathrm{H}$ stretching. Unfortunately, Raman spectroscopy did not show any band from CAM in PEDOT/CAM NPs due to the strong absorbance of PEDOT chains. The recorded spectra and the corresponding discussion is provided in the Supporting Information (Figure S4). On the other hand, Figure 4d, which compares the UV spectra of CAM, PEDOT NPs and PEDOT/CAM NPs, confirms the lack of covalent bonding between drug and the polymers chains. Furthermore, the spectra do not reveal the formation of CAM $\cdots$ PEDOT $\pi-\pi$ stacking interactions, supporting the fact that the interactions between the two species are mainly electrostatic.

Quantitative data on the composition of PEDOT/CAM NPs were obtained by X-ray photoelectron spectroscopy (XPS). The EDOT:CAM ratio was determined using the atomic percentage composition, which is shown in Table 1. Considering that there is one sulphur atom per EDOT repeat unit and two nitrogen atoms per CAM molecule (Scheme 1), the S $2 p$ / $\mathrm{N}$ 1s ratio indicates $\sim 4$ EDOT units per CAM molecule. This value is consistent with the one derived from the $\mathrm{S} 2 \mathrm{p} / \mathrm{Cl} 2 \mathrm{p}$ ratio (two chlorine atoms per CAM molecule), which is $\sim 3$ EDOT unit per CAM molecule.

More detailed information regarding the chemical bonds present on the sample was extracted from the peak's deconvolution, as shown in Figures 2 and S5. The nitrogen peak, which is completely absent for PEDOT NPs (Figure 2a), is centered at $399.8 \mathrm{eV}$ for PEDOT/CAM NPs (Figure 2b) and has been attributed to $\mathrm{N}-\mathrm{H}$ and $\mathrm{C}-\mathrm{N}$ from CAM. Similarly, the $\mathrm{Cl} 2 \mathrm{p}$ peak only appears for PEDOT/CAM NPs (Figure 2c-d), showing a spin-split couple, Cl 2p3/2 and $\mathrm{Cl} 2 \mathrm{p} 1 / 2$, at $198.4 \mathrm{eV}$ and $200 \mathrm{eV}$, respectively. This peak corresponds to the $\mathrm{C}-\mathrm{Cl}$ present also in CAM.

Figure S5 displays the high-resolution XPS spectrum in the C 1s, S 2p and O 1s regions for PEDOT and PEDOT/CAM NPs. Deconvolution of the C 1s peak (Figure S5a-b) led to five 


\section{WILEY-VCH}

Gaussian curves. Of these, four peaks have been attributed to bonds from PEDOT chains:

$\mathrm{C}-\mathrm{C}$ and $\mathrm{C}-\mathrm{H}$ bonds of PEDOT chains and the surfactant molecules $(284.8 \mathrm{eV}) ; \mathrm{C}-\mathrm{S}$ bonds

of thiophene ring $(286.4 \mathrm{eV})$; the $\mathrm{C}-\mathrm{O}$ bonds of the fused dioxane ring $(288.0 \mathrm{eV})$; and the $\pi \cdots \pi^{*}$ shake up due to the thiophene ring $(289.15 \mathrm{eV}) .^{[21]}$ The deconvoluted small peak centered at $283 \mathrm{eV}$ has been associated to the $\mathrm{Al}-\mathrm{C}$ (carbide) bond from the XPS pin substrate. On the other hand, the high resolution XPS of the $\mathrm{S} 2 \mathrm{p}$ region of PEDOT and PEDOT NPs (Figure S5c-d) show the spin-split sulfur coupling S2 $\mathrm{p}_{3 / 2}$ and $\mathrm{S} 2 \mathrm{p}_{1 / 2}$, with a separation of 1.2 $\mathrm{eV}$, for the $\mathrm{C}-\mathrm{S}-\mathrm{C}$ bond of the thiophene ring (162.7 and $163.9 \mathrm{eV}$, respectively) in PEDOT chains, ${ }^{[22]}$, and the $\mathrm{SO}_{3}{ }^{-}$of DBSA (167.6 eV and $168.8 \mathrm{eV}$, respectively). ${ }^{[21 \mathrm{a}, 23]}$ The $\mathrm{O} 1 \mathrm{~s}$ signal consists of three components (Figure S5e-f). The first is related to water remaining on the sample $(534.0 \mathrm{eV}),{ }^{[24]}$ while the peaks at 532.1 and $530.8 \mathrm{eV}$ correspond to the $\mathrm{C}-\mathrm{O}-\mathrm{C}$ of the fused dioxane ring and the $\mathrm{SO}_{3}{ }^{-}$of DBSA dopant molecules. ${ }^{[25]}$

\subsection{Morphology of PEDOT and PEDOT/CAM NPs}

Scanning electron microscopy (SEM) and atomic force microscopy (AFM) were employed to visualize and characterize the morphology of the prepared NPs. SEM micrographs of PEDOT and PEDOT/CAM NPs are displayed in Figure 3a (top and bottom, respectively). Both types of NPs present a well-defined spherical shape. The average diameter, as determined by DLS, is not altered by the encapsulated drug (i.e. $118 \pm 16$ and $121 \pm 13 \mathrm{~nm}$ for PEDOT and PEDOT/CAM, respectively). The resulting histograms, which are included in Figure 3a, evidence that the size distribution is very narrow, in perfect correlation with the low polydispersity values obtained by DLS.

These results were confirmed by AFM, both 3D topographic and 2D phase images being displayed in Figure 3b. The diameter of PEDOT and PEDOT/CAM NPs, as determined from AFM images, is $139 \pm 19$ and $134 \pm 23 \mathrm{~nm}$, respectively. This noticeable similarity is in sharp contrast with the results reported for PEDOT/CUR and PEDOT/PIP NPs, which exhibited a 
diameter much higher than PEDOT NPs (i.e. $~ 150 \%$ and $\sim 260 \%$ increment, respectively). ${ }^{[18]}$ Considering that molecular sizes of CAM, CUR and PIP are similar, these observations suggest that the interactions between CAM and PEDOT chains are radically different from those formed by CUR and PIP and, therefore, the release mechanism is also expected to be different. On the other hand, after the loading of PPEPs, which are larger than CAM, PEDOT NPs resulted in an increase in diameter of only about $23 \%-37 \%{ }^{[19]}$

\subsection{CAM release}

The release of CAM from the dialysis buttons containing PEDOT/CAM NPs was studied by UV-Vis in media with different hydrophilicities: phosphate buffered saline (PBS) solution alone, PBS with $10 \%$ ethanol (PBS:EtOH 90:10), and PBS with $70 \%$ ethanol (PBS:EtOH 30:70). In a preliminary release assay, which was performed only in PBS (not shown), we observed that cumulative release of CAM in such hydrophilic medium is very slow, reaching a value of only $19 \%$ after 82 days (not shown). Then, a second release experiment was designed as described in the Methods section (i.e. decreasing the hydrophilicity of the medium every week by replacing PBS alone by ethanol-containing PBS solutions). The release profile, which is displayed in Figure 4, indicates that the release during the first two weeks was very slow. This is due to the poor affinity of the hydrophobic drug by PBS and PBS:EtOH 90:10, which does not compensate the strength of the interactions between CAM molecules and oxidized PEDOT chains. However, when the latter solution was replaced with PBS:EtOH 30:70, the release of CAM increased significantly, reaching $100 \%$ in only six days (Figure S5). In summary, CAM has a great affinity towards EtOH molecules and, indeed, is very soluble in this solvent (up to $50 \mathrm{mg} / \mathrm{mL}$ ), whereas the affinity towards water molecules is poor (this drug in water is only slightly soluble in water, up to $2.5 \mathrm{mg} / \mathrm{mL}$ ) Accordingly, CAM $\cdots$ water interactions cannot compete with CAM $\cdots$ CAM and CAM $\cdots$ PEDOT interactions to favour the release of the encapsulated drug. On the other hand, it should be 


\section{WILEY-VCH}

emphasized that, although the release of CAM in PBS is low, it is enough to inhibit the bacterial growth and, in addition, can be increased by electrical stimulation and by the alteration of the composition through the addition of nutrients (as proved in next sections). In recent studies, antimicrobial CAM was encapsulated in non-electroresponsive polymeric carriers for release. ${ }^{[26-28]}$ For example, CAM-loaded polycaprolactone (PCL) nanofibers and, especially, PCL- polyethylene oxide (PEO) and microfiber exhibited a very fast release in PBS, reaching in $1 \mathrm{~h}$ more than $30 \%$ and $90 \%$, respectively. ${ }^{[26]}$ The difference between such two systems was attributed to the hydrophobic and hydrophilic nature of PCL and PCL-PEO fibers, respectively. In any case, the strength of the interaction between CAM and such polymers was significantly weaker than with PEDOT. Similarly, the release of CAM loaded in poly(vinyl alcohol)/sodium alginate hydrogels was very high, independently of the ratio between such two polymers, reaching more than $50 \%$ in less than 3 h. ${ }^{[27]}$ Similarly, CAM was easily released from both loaded amorphous calcium phosphate (ACP) and hydroxyapatite nanoparticles in the simple physiological PBS medium. ${ }^{[28]}$ Overall, these observations support the strength of CAM $\cdots$ PEDOT interactions.

\subsection{Electrochemical properties}

The main objective of this work is to design an implantable therapeutic device to control the progression of bacterial infections by inhibiting and, at the same time, monitoring bacterial growth. Therefore, it is necessary to evaluate the response of PEDOT and PEDOT/CAM NPs to electrical stimuli. It is worth noting that the engineered application requires stable NPs without excessive drug release when monitoring by $\mathrm{CV}$ (cyclic voltammetry). A broad potential window (from -0.50 to $1.40 \mathrm{~V}$ ) was used to record the cyclic voltammogram of SPCE-PEDOT in a solution of PBS $1 \times$ with CAM as supporting electrolyte (Figure 5a). CAM exhibits a well-defined cathodic peak at around $-0.4 \mathrm{~V}$ that corresponds to the reduction of the nitro group to an amino group, following a four electron and four proton 


\section{WILEY-VCH}

transfer mechanism. ${ }^{[29]}$ The increase of the anodic current at voltages higher than $1 \mathrm{~V}$ has been attributed to the production of oxygen and protons due to the oxidation of water molecules.

Cyclic voltammograms recorded for the bare electrode (SPCE), SPCE coated with PEDOT/CAM NPs (SPCE-PEDOT/CAM) and SPCE coated with PEDOT NPs (SPCEPEDOT) in PBS are compared in Figure 5b. The area of the voltammogram increases considerably when the carbon SPCE is coated with PEDOT or PEDOT/CAM NPs, evidencing an enhancement of the capacitive behaviour. However, no difference was observed between PEDOT NPs and PEDOT/CAM NPs, suggesting that the amount of CAM is very low (i.e. the drug is released during the potential scan) or that the signal of the drug overlaps with the one of PEDOT NPs. In order to get a deeper understanding on this feature, the effect of the electrical voltage on the drug retention was analysed. More specifically, the release of CAM was investigated applying continuous stimulation by chronoamperometry (CA), keeping constant voltage at $1.00 \mathrm{~V}$, and by $\mathrm{CV}$, ramping the voltage from -0.50 to 0.50 V. In both cases, CA and CV, stimuli were applied for 5, 15 and 30 min. Control experiments (CRTL) were also performed using the same periods of time but without electrical stimulation. Results displayed in Figure 5c indicate that the amount of CAM released from PEDOT/CAM is only $\sim 30 \%$ and $\sim 20 \%$ higher for CA and CV, respectively, than for the CTRL, meaning that PEDOT/CAM NPs are very stable. Furthermore, comparison of the cyclic voltammograms recorded between -0.50 and $0.50 \mathrm{~V}$ after $5,15 \mathrm{~min}$ and $30 \mathrm{~min}$ of $\mathrm{CV}$ stimulation (Figure $5 \mathrm{~d}$ ) reveals a slight decrease of the current density at the voltage where CAM is reduced. This phenomenon has been attributed to the small amount of drug released.

\subsection{Evaluation of cytotoxicity}

As the proposed application for the PEDOT/CAM NPs is their therapeutic utilization as antibiotic carriers and bacterial growth monitoring, evaluation of the biocompatibility is 


\section{WILEY-VCH}

essential. The influence of the system on in vitro cell viability was investigated using the MTT [3-(4, 5-dimethylthiazol-2-yl)-2, 5-diphenyltetrazolium bromide] assay (see Supporting Information). Different CAM concentrations were exposed for $24 \mathrm{~h}$ to cultures of commercial human bone osteosarcoma MG-63 cell line. Figure 6a shows that the half-inhibitory concentration (IC50) of free CAM for MG-63 is around $350 \mu \mathrm{g} / \mathrm{mL}$, evidencing that the tolerance of the cells to this drug is much higher than that found for other drugs, such as CUR (with a IC50 ranging from 10 to $20 \mu \mathrm{g} / \mathrm{mL}$, depending on the cell line ${ }^{[18]}$ ). The biocompatibility of PEDOT and PEDOT/CAM NPs was also evaluated using the MG-63 cell line. Figure $6 \mathrm{~b}$ shows that cells have a very high tolerance to PEDOT NPs without exhibiting any reduction in cell viability. Instead, the cell viability clearly decreases when high concentrations of PEDOT/CAM NPs are used. In any case, the IC50 of free CAM and PEDOT/CAM NPs for eukaryotic cells is much higher than the dose of antibiotic required to inhibit the growth of bacteria growth, as in demonstrated below. Thus, antibiotics are much more active against bacteria than against eukaryotic cells, allowing their utilization for the treatment of bacterial infections without damage the eukaryotic human cells.

\subsection{Bactericidal activity}

In order to confirm that the active conformation of CAM was not altered during the synthesis of PEDOT/CAM NPs, the activity of the loaded antibiotic was tested against a representative Gram-negative bacterium (E. coli) and a Gram-positive bacterium (Streptococcus sanguinis, hereafter S. sanguinis). The observed activity was compared with that of the controls, which were free CAM, free nalidixic acid (NAX; a well-known synthetic quinolone antibiotic) and medium alone. For this purpose, we used the disk diffusion test, which provides qualitative evaluation of the susceptibility of bacteria to the molecules diffusing from the disk.

Results showed in Figure 7a-b indicate that free CAM and free NAX are the most effective for inhibiting bacterial growth, followed by PEDOT/CAM NPs. Moreover, the bactericidal 


\section{WILEY-VCH}

activity was slightly higher for E. coli than for S. sanguinis. Instead, PEDOT NPs do not show any antibacterial behavior. On the other hand, Figure $7 \mathrm{c}-\mathrm{d}$ plots the relative growth rate of the S. sanguinis and E. coli when different amounts of PEDOT/CAM (i.e. volumes of 5, 25 and $50 \mu \mathrm{L}$ from a $10 \mathrm{mg} / \mathrm{mL}$ suspension), free CAM, PEDOT NPs $(50 \mu \mathrm{L}$ of a $10 \mathrm{mg} / \mathrm{mL}$ suspension) are added to bacteria cultures. The growth rate in the LB medium without any kind of NPs or antibiotic was taken as the control (CTRL). Addition of free CAM to the medium causes a quick and effective inhibition of bacterial growth, whereas the relative growth obtained upon the addition of PEDOT NPs is similar to that of the CTRL.

Consistently with images displayed in Figure 7a-b, the difference between the absorbance of samples incubated with PEDOT/CAM NPs and the control after $48 \mathrm{~h}$ is higher for the E. coli than for the S. sanguinis. Besides, the inhibition of the bacterial growth caused by the addition of PEDOT/CAM increases with the concentration of antibiotic-loaded NPs in the medium, the most effective inhibition being observed for the volume of $50 \mu \mathrm{L}$.

\subsection{Monitoring of bacterial growth}

The performance of PEDOT and PEDOT/CAM NPs for real-time electrochemical monitoring of bacterial growth or inhibition is discussed in this section. With the aim of orienting this sensing and release device toward clinical applications, PEDOT and PEDOT/CAM NPs were fixed on SPCEs and Dulbecco's Modified Eagle's Medium (DMEM) with the corresponding bacteria was used as electrolytic media. The electrolytic chambers were kept on an incubator at $37^{\circ} \mathrm{C}$ under mild-agitation and the growth of bacteria was followed by examining the electrochemical response at different times, which ranged from $0 \mathrm{~h}$ (just when the bacteria are introduced into the cell chamber) to $24 \mathrm{~h}$. Figure 8a displays the voltammetric response of the sensor to the culture medium without bacteria at different incubation times. As shown, the variation in the area and the position of the peaks in the voltammograms recorded at different times is negligible $(<1 \%)$. Furthermore, the surface of the electrode was not damaged by the 


\section{WILEY-VCH}

cell culture medium. This is proved in Figure 8b-c, which display a representative SEM micrographs of PEDOT NPs coated electrodes as prepared and after $24 \mathrm{~h}$ in $\mathrm{NaHCO}_{3}-$ supplemented DMEM without bacteria.

Cyclic voltammograms recorded during the S. sanguinis growth on PEDOT NPs-containing electrodes are displayed in Figure 9a. The small anodic peak at around $0.6 \mathrm{~V}$ detected at $\mathrm{t}=0$ $\mathrm{h}$ has been associated to the phenol red present in the cell culture media. Phenol red, which is one of the most common $\mathrm{pH}$ indicators and a weak acid, exhibits redox properties through the ionizable part of the quinone methide. ${ }^{[30]}$ The current density of such oxidation peak increases with the incubation time while the potential shifts to higher values, which has been attributed to a reduction of the $\mathrm{pH}$. Thus, bacterial respiration pumps out protons, causing the acidification of the medium with increasing time. Qiao et al. ${ }^{[31]}$ reported that the anodic peak related with the oxidation of bacterial metabolites shifts to higher peak potential values with decreasing $\mathrm{pH}$. On the other hand, the increment of current density at the potential peak with the incubation time evidences the presence of other molecules that are oxidizing. This has been related to the increasing concentration of NADH in the electrolytic media due to the bacteria respiration, which oxidizes at around $0.6 \mathrm{~V} \cdot{ }^{[10]}$ Another remarkable feature is that the area of the voltammograms increases with the incubation time, which is consistent with previous studies based on the utilization of the metabolism of living microorganisms to generate renewable electrical energy flux. ${ }^{[32]}$

Comparison with the voltammetric response of PEDOT/CAM NPs against S. sanguinis, which is shown in Figure 9b, reveals important differences with respect to PEDOT NPs. More specifically, the peak at around $0.6 \mathrm{~V}$ does not shift and the area of the voltammograms does not increase with the incubation time. This has been attributed to the inhibition of bacterial growth by released CAM, as was corroborated by visualizing the electrode surface after the $24 \mathrm{~h}$ of culture. The release of CAM after such period of time ( $24 \mathrm{~h})$ was determined to be of $14.8 \pm 0.8 \mu \mathrm{g} / \mathrm{mL}$, which corresponds to $2.2 \% \pm 0.1$ of the loaded drug. Comparison with the 


\section{WILEY-VCH}

release profile obtained in PBS (Figure 4) indicates that the culture medium $\left(\mathrm{NaHCO}_{3}\right.$ supplemented DMEM), which contains different nutrients (e.g. salts, amino acids and vitamins) that affects the chemical and physical properties of the fluid, significantly enhances the CAM release (from $0.9 \%$ in PBS to $2.2 \%$ in the culture medium). The original DMEM formula composition contains $1000 \mathrm{mg} / \mathrm{L}$ of glucose and was first reported for culturing embryonic mouse cells. A further alterati significantly higher that obtained for PBS (Figure 4), value is significantly higher that SEM micrographs of PEDOT and PEDOT/CAM NPs electrode surfaces are shown in Figure 9c-d, respectively. The number of bacteria, which are artificially highlighted in orange for easy viewing, is much higher for PEDOT than for PEDOT/CAM.

In order to quantify differences in the bacteria content after $24 \mathrm{~h}, 50 \mu \mathrm{L}$ were taken from electrolytic cells containing only culture medium, culture medium with bacteria, and bacteria cultured in the presence of PEDOT and PEDOT/CAM NPs. The observed bacterial growth, which was determined by measuring the absorbance at $600 \mathrm{~nm}$, is represented in Figure 9e. It is worth noting that, after $24 \mathrm{~h}$, the bacterial growth on PEDOT NPs coated electrodes equalled that of the control group, while it was lower than $15 \%$ for PEDOT/CAM NPs. Figure 9 f plots the change in current density of the main peak at around $0.6 \mathrm{~V}$ for the different time intervals relative to the one at $0 \mathrm{~h}(\Delta j)$. It is worth remarking that $\Delta j$ correlates with bacterial growth. Thus, $\Delta j$ is smaller than $6 \mu \mathrm{A} / \mathrm{cm}^{2}$ when there are no bacteria or when CAM is present in the electrode coating, whereas $\Delta j$ reaches a value of up to $\sim 16 \mu \mathrm{A} / \mathrm{cm}^{2}$ for SPCEs coated with PEDOT NPs (which supports normal bacterial growth as CAM is not present). In order to prove that the detection is independent of the type of bacteria, the same set of experiments were conducted for E. coli (Figure S7). Interestingly, we observed the same behaviour by CV. The area of the voltammograms recorded for PEDOT NPs increased with the incubation time, while this did not happen for PEDOT/CAM NPs (Figure S7a-b). These 


\section{WILEY-VCH}

observations are consistent with SEM micrographs, which display groups of E. coli only on the surface of PEDOT NPs-containing electrodes (Figure S7c-d). Measurement of the absorbance at $600 \mathrm{~nm}$ after $24 \mathrm{~h}$ reflected that the relative growth of bacteria was practically zero for PEDOT/CAM NPs (Figure S7e), indicating that the CAM treatment is very effective for E. coli. Also, $\Delta j$ is lower than $2 \mu \mathrm{A} / \mathrm{cm}^{2}$ in the absence of bacteria and for bacteria cultured on PEDOT/CAM NPs, whereas a value close to $26 \mu \mathrm{A} / \mathrm{cm}^{2}$ was reached for PEDOT NPs (Figure S7f).

Results related with bacteria growth and inhibition in the presence of PEDOT and PEDOT/CAM NPs, respectively, are supported by the color of the phenol red-containing medium after $24 \mathrm{~h}$ (Figure S8). Thus, the medium in contact with PEDOT NPs exhibited a yellowish color, which was attributed to the acidification induced by the bacterial growth, while the medium in contact with PEDOT/CAM was pink, reflecting a basic $\mathrm{pH}$. Overall, results discussed in this section clearly demonstrate that SPCEs coated with PEDOT and PEDOT/CAM NPs can monitor the bacterial growth and the inhibition of the bacterial growth, respectively, in real time.

\subsection{NADH detection}

The increase in current density in the $\sim 0.6 \mathrm{~V}$ potential region, as displayed in Figures $9 \mathrm{a}$ and S7a, was related in previous work with the increment of NADH in the bacteria culture medium. ${ }^{[10]}$ Our approach was based on the fact that aerobic respiration reactions in eukaryotic cells occur in the mitochondria, whose double membrane is impermeable to $\mathrm{NADH}$ and NAD+. Therefore, the NADH level in eukaryotic level is restricted to the cytosolic pool. Instead, the respiration of bacteria occurs in the cytosol or on the inner surfaces of the cell membrane, which is permeable to NADH and NAD + and allows their migration to the extracellular space. This inspired us to use the oxidation of extracellular 
$\mathrm{NADH}$ to NAD+ as target for the detection of bacterial growth without interference coming from the proliferation of eukaryotic cells.

In this section we prove such relationship by measuring the influence of increasing NADH concentrations on cyclic voltammograms recorded for PEDOT NPs coated SPCEs using DMEM (pH 8.5) as electrolytic medium. Figure 10a compares the cyclic voltammograms obtained using a NADH concentration comprised within 0 and $2 \mathrm{mM}$. In the absence of NADH $(0 \mathrm{mM})$, a small peak is detected at $0.6 \mathrm{~V}$ that has been attributed to the redox properties of the phenol red present in the medium, as-discussed above. The addition of $\mathrm{NADH}$ with concentrations ranging from $250 \mu \mathrm{M}$ to $2 \mathrm{mM}$ resulted in a significant enhancement of the current density during anodic screening. The NADH oxidation peak is very broad and clearly overlaps with the small peak initially encountered at $0.6 \mathrm{~V}$ (i.e. the phenol red oxidation peak observed in the absence of NADH). Moreover, Figure 10b demonstrates a linear relationship between the relative increment of the current density and the concentration of NADH.

Another aspect to consider is that, during bacterial growth, the medium undergoes an acidification process. Accordingly, cyclic voltammograms were also recorded using DMEM at pH 6 as electrolytic medium. Figure 10c shows that the well-defined peak coming from the medium, which is centered at $0.62 \mathrm{~V}$, increases with the NADH concentration. Although the potential anodic peak shifts a little bit towards higher values with increasing NADH concentration, the relative increment of the current density exhibits a linear variation (Figure 10d). Overall, this section supports the important role that NADH, one of the main redox compounds produced by microbial metabolism and released to the medium, plays for in situ monitoring the bacterial growth using $\mathrm{CV}$.

\section{Conclusion}




\section{WILEY-VCH}

In this work, a simple and highly sensitive electrode consisting of CAM-loaded PEDOT NPs has been developed to monitor the inhibition of bacterial growth. More specifically, we have engineered a nanotheranostic system able to sense the infection progression while releasing the antibiotic, inhibiting the E. coli and S. sanguinis growth. After characterization, the drug release was evaluated in hydrophilic media. Although a slow drug release was observed, independently of the presence or not of electrical stimuli (i.e. $14.8 \pm 0.1 \mu \mathrm{g} / \mathrm{mL}$ of CAM after $24 \mathrm{~h}$ in the cell culture medium), the bacterial growth inhibition efficacy of the drug was clearly maintained after its incorporation to the polymeric NPs. Furthermore, we proved that PEDOT and PEDOT/CAM NPs can monitor the increase and inhibition of the bacterial growth, respectively, using $\mathrm{CV}$ by detecting the oxidation of $\mathrm{NADH}$ at $0.6 \mathrm{~V}$, which diffuses to the extracellular medium after being produced by the bacterial metabolism. This versatility suggests that the developed nanoparticles are a promising theranostic system for the treatment and control of bacterial infections. Thus, we envisage a future with prostheses, implants and other medical devices coated with drug-loaded theranostic nanoparticles informing and reporting pathogenic growth in real time.

\section{Methods}

Synthesis and characterization. The synthesis and characterization of PEDOT and PEDOT/CAM NPs, respectively, are extensively described in the Supporting Information. In brief, both PEDOT and PEDOT/CAM NPs were prepared by oxidative polymerization in dodecylbenzene sulfonic acid (DBSA) micellar solutions. DBSA was used to act as both surfactant to form micelles and, thus, solubilize the 3,4-ethylenedioxythiophene (EDOT) monomer, and as dopant agent. DBSA was used in combination with ammonium persulfate (APS), which acted as an oxidant. The loading of CAM was performed in situ by adding an ethanol drug solution to the reaction medium, as described in the Supporting Information. The loading capacity (LC, in \%) was determined using the procedure described in the Supporting 


\section{WILEY-VCH}

Information (Equation S1). Unloaded and CAM-loaded NPs were characterized using SEM, AFM, DLS, both FTIR and Raman spectroscopies, and XPS. Details are provided in the Supporting Information.

Drug release. $25 \mu \mathrm{L}$ of PEDOT/CAM NPs $(10 \mathrm{mg} / \mathrm{mL})$ were deposited into dialysis buttons, covered with a $3.5 \mathrm{kDa}$ MWCO (Molecular Weight Cut-Off) dialysis membrane, immersed in $1.5 \mathrm{~mL}$ of PBS solution ( $\mathrm{pH} 7.4$ ), and kept in a shaker at $37^{\circ} \mathrm{C}$ at $80 \mathrm{rpm}$. Each day all the immersion solution was taken out to quantify the released drug and the solution was replaced with $1.5 \mathrm{~mL}$ of new medium. The release process in PBS was evaluated for more than 80 days. After this, a second experiment in which the release medium was changed every week from hydrophilic to hydrophobic, was conducted for three weeks. More specifically, the release medium for the first, second and third week was PBS, PBS:EtOH 90:10, and PBS:EtOH 30:70, respectively, the latter allowing the complete drug release. All the results were normalized by the total amount of drug encapsulated within the NPs or used as a free molecule in order compare their kinetics. The amount of released drug was evaluated by UV spectroscopy using a Cary100 UV-Vis spectrophotometer controlled by the UVProbe 2.31 software. Calibration curves were obtained by plotting the absorbance measured at $280 \mathrm{~nm}$ against CAM concentration (Figure S2). Release experiments were repeated three times and the averages were plotted.

Effects of voltage and time on drug release. Washed PEDOT/CAM NPs were re-suspended in milli-Q water to a final concentration of $10 \mathrm{mg} / \mathrm{mL}$. Then, a total of $15 \mu \mathrm{L}$ of the resulting solution were placed on SPCEs in three rounds ( $5 \mu \mathrm{L}$ each time) and dried under hood after each round. Dried NPs were covered with $5 \mu \mathrm{L}$ of chitosan solution $(20 \mathrm{mg} / \mathrm{mL}$ chitosan in $0.1 \mathrm{M} \mathrm{HCl}$ ) and dried again. The chitosan layer formed after drying was used to fix the NPs, avoiding detachment. A three-electrode configuration was used: the SPCE, SPCEPEDOT/CAM or SPCE/PEDOT worked as a working electrode; a platinum wire as counter 


\section{WILEY-VCH}

electrode; and $\mathrm{Ag} \mid \mathrm{AgCl}$ as reference electrode. $1.5 \mathrm{~mL}$ of PBS $1 \times$ was used as electrolytic medium.

The appropriate voltage was applied for a fixed time. After electrical stimulation, the medium was kept to determine the drug concentration and replaced with new PBS. The absorbance was read at $280 \mathrm{~nm}$. The influence of the time was evaluated by CA using a voltage of $+1.0 \mathrm{~V}$ and by $\mathrm{CV}$ using a potential window from $-0.50 \mathrm{~V}$ to $+0.50 \mathrm{~V}$ during 5,15 and $30 \mathrm{~min}$. A control experiment was performed in the absence of the stimulus. All the measures were repeated at least three times and the average with the standard deviation plotted on the graphs. Cyclic voltammetry. CV studies were conducted with an Autolab PGSTAT302N Galvanostat equipped with the ECD module (Ecochimie, The Netherlands). Measurements were performed on $15 \mu \mathrm{L}$ of $10 \mathrm{mg} / \mathrm{mL}$ NPs solution, which were fixed on a SPCE with chitosan, as described previously. All electrochemical assays were performed using a three-electrode one compartment cell at room temperature. The cell was filled with $1.5 \mathrm{~mL}$ of PBS $1 \times$ as a supporting electrolyte. A SPCE coated with NPs (i.e. SPCE-PEDOT or SPCE-PEDOT/CAM) was used as the working electrode, platinum wire as the counter electrode, while an $\mathrm{Ag} \mid \mathrm{AgCl}$ electrode containing $\mathrm{KCl}$ saturated aqueous solution was the reference electrode (offset potential versus the standard hydrogen electrode, $\mathrm{E}^{\circ}=0.222 \mathrm{~V}$ at $25^{\circ} \mathrm{C}$ ). Oxidation-reduction cycles were registered within the potential range of -1.5 to $+1.0 \mathrm{~V}$ at different scan rates. Bacterial growth detection. Bacterial growth was monitored in real time on the electrochemical cell by CV. Voltammograms of coated SPCEs in the culture medium, which was $\mathrm{NaHCO}_{3}$-supplemented DMEM, were recorded at different incubation times $(0,2,4,6,8$ and $24 \mathrm{~h}$ ) for PEDOT and PEDOT/CAM NPs in the presence and absence of $E$. coli and $S$. sanguinis. The initial and final potentials were $-0.20 \mathrm{~V}$, while the reversal potential and the scan rate were $0.80 \mathrm{~V}$ and $100 \mathrm{mV} / \mathrm{s}$, respectively. For comparison with the results obtained by $\mathrm{CV}$, the relative bacterial growth was evaluated through the variation of the absorbance at 


\section{WILEY-VCH}

$600 \mathrm{~nm}$ after $24 \mathrm{~h}$ of culture. According to the McFarland standard, one unity of absorbance at $600 \mathrm{~nm}$ was associated to $10^{8} \mathrm{CFU} \mathrm{mL}$.

NADH detection. NADH was detected by $\mathrm{CV}$ using SPCE-PEDOT. Measures were performed using different $\mathrm{NADH}$ concentrations at $\mathrm{pH} 8.5$ and 6 in $\mathrm{NaHCO}_{3}$-supplemented DMEM (i.e. culture medium). The initial and final potentials were $-0.20 \mathrm{~V}$, while the reversal potential and the scan rate were $0.80 \mathrm{~V}$ and $100 \mathrm{mV} / \mathrm{s}$, respectively.

CAM antibacterial activity test. The bactericidal activity of the loaded drug was tested with $E$. coli and S. sanguinis. First, $1 \mathrm{~mL}$ of an overnight culture (grown for $16 \mathrm{~h}$ ), was added to $5 \mathrm{~mL}$ of the Lysogeny broth (LB) medium. Bacteria were seeded on LB agar plates and four paper discs impregnated with the different samples deposited on top. Three of such discs contained $25 \mu \mathrm{L}$ of $10 \mathrm{mg} / \mathrm{mL}$ PEDOT/CAM NPs, $10 \mathrm{mg} / \mathrm{mL}$ PEDOT NPs or $2 \mathrm{mg} / \mathrm{mL}$ CAM dissolved in ethanol, respectively, while the fourth disc was impregnated with NAX, as a control. The effect of the drugs on bacterial growth was evaluated after incubation at $37^{\circ} \mathrm{C}$ for $24 \mathrm{~h}$. The test was carried out in triplicate.

Bacterial growth curves. The bactericidal activity of loaded CAM was tested during E. coli and $S$. sanguinis growth. For this purpose, six $15 \mathrm{~mL}$ polystyrene tubes were filled with $5 \mathrm{~mL}$ of LB medium and, subsequently, $10 \mu \mathrm{L}$ of concentrated bacteria $\left(10^{8} \mathrm{CFU} / \mathrm{mL}\right)$ were added, reaching $1 \times 10^{3} \mathrm{CFU}$ per tube (estimated by the absorbance at $600 \mathrm{~nm}$ ). 0 (blank), 5, 25 and 50 $\mu \mathrm{L}$ of $10 \mathrm{mg} / \mathrm{mL}$ PEDOT/CAM NPs, $50 \mu \mathrm{L}$ of PEDOT NPs or $25 \mu \mathrm{L}$ of $2 \mathrm{mg} / \mathrm{mL}$ CAM solution in ethanol together with $100 \mu \mathrm{L}$ of fresh LB medium were placed in $3.5 \mathrm{kDa} \mathrm{MWCO}$ dialysis bags. Each dialysis bag was immersed in the previously described polystyrene tubes. All tubes were kept in an incubator at $37^{\circ} \mathrm{C}$ and $80 \mathrm{rpm} .40 \mu \mathrm{L}$ of medium were taken from each tube after $0,2,4,6,24$ and $48 \mathrm{~h}$ and the absorbance checked by UV at $600 \mathrm{~nm}$ to estimate bacterial growth. Bacterial growth in the tube with only culture medium (i.e. in the absence of NPs or drug) was considered as the maximum growth (control) and it was used to 


\section{WILEY-VCH}

calculate the relative growth of the bacteria in the presence of the different samples. All assays were conducted in triplicate and the values averaged.

Statistical Analysis. The data of the experiments were presented as mean \pm standard deviation (SD). Multiple comparisons among groups (cell viability) were determined using one-way ANOVA analysis; ${ }^{*}, \mathrm{p}<0.05 ; * *, \mathrm{p}<0.01 ; * * *, \mathrm{p}<0.001$ represented a significant difference.

\section{Supporting Information}

Supporting Information is available from the Wiley Online Library or from the author.

\section{Acknowledgements}

Authors acknowledge MINECO/FEDER (RTI2018-098951-B-I00 and RTI2018-101827-BI00), the Agència de Gestió d'Ajuts Universitaris i de Recerca (2017SGR359) and B. Braun Surgical, S.A. company for financial support. Support for the research of C.A. was received through the prize "ICREA Academia" for excellence in research funded by the Generalitat de Catalunya.

Received: ((will be filled in by the editorial staff))

Revised: ((will be filled in by the editorial staff)) Published online: ((will be filled in by the editorial staff))

\section{References}

[1] a) M. Amiri, A. Bezaatpour, H. Jafari, R. Boukherroub, ACS Sensors 2018, 3, 1059; b) J. Monzó, I. Insua, F. Fernandez-Trillo, P. Rodriguez, Analyst 2015, 140, 7116; c) R. Cagliani, M. Sironi, Int. J. Evol. Biol. 2013, 204240.

[2] a) B. Aslam, W. Wang, M. I. Arshad, M. Khurshid, S. Muzammil, M. H. Rasool, M. A. Nisar, R. F. Alvi, M. A. Aslam, M. U. Oamar, M. K. F. Salamat, Z. Baloch, Infect. Drug Resist. 2018, 11, 1645; b) J. M. Pogue, K. S. Kaye, D. A. Cohen, D. Marchaim, Clin. Microbiol. Infect. 2015, 21, 302.

[3] O. Simoska, K. J. Stevenson, Analyst 2019, 144, 6461.

[4] O. Lazcka, F. J. del Campo, F. X. Muñoz, Biosens. Bioelectron. 2007, 22, 1205.

[5] a) L. Wang, Z. Lou, K. Jiang, G. Shen, Adv. Intell. Syst. 2019, 1, 1900040; b) A. E.

Rochford, A. Carnicer-Lombarte, V. F. Curto, G. G. Malliaras, D. G. Barone, Adv. Mater. 


\section{WILEY-VCH}

2020, 32, 1903182; c) J. H. Park, H. E. Lee, C. K. Jeong, D. H. Kim, S. K. Hong, K.-II. Park, K. J. Lee, Nano Energy 2019, 56, 531.

[6] A. Buzid, F. J. Reen, V. K. Langsi, E. O. Muimhneacháin, F. O’Gara, G. P.

McGlacken, J. H. T. Luong, J. D. Glennon, ChemElectroChem 2017, 4, 533.

[7] H. J. Sismaet, A. J. Pinto, E. D. Goluch, Biosens. Bioelectron. 2017, 97, 65.

[8] M. Sedki, R. Y. A. Hassan, A. Hefnawy, I. M. El-Sherbiny, Sensors Actuators, B Chem. 2017, 252, 191.

[9] K. Butina, S. Löffler, M. Rhen, A. Richter-Dahlfors, Sensors Actuators, B Chem. 2019, $297,126703$.

[10] B. G. Molina, L. J. Del Valle, P. Turon, E. Armelin, C. Alemán, J. Phys. Chem. C 2019, 123, 22181.

[11] a) A. Okamoto, S. Kalathil, X. Deng, K. Hashimoto, R. Nakamura, K. Nealson, Sci. Rep. 2014, 4, 1; b) b) A. Okamoto, K. Hashimoto, K. H. Nealson, R. Nakamura, Proc. Natl. Acad. Sci. U. S. A. 2013, 110, 7856.

[12] a) D. Li, P. Lv, L. Fan, Y. Huang, F. Yang, X. Mei, D. Wu, Biomater. Sci. 2017, 5, 2337; b) D. King, S. McGinty, J. Control. Release 2016, 239, 49; c) F. Tan, Y. Zhu, Z. Ma, M. Al-Rubeai, Acta Biomater. 2020, 108, 46; d) J. D. Caplin, A. J. García, Acta Biomater. 2019, $93,2$.

[13] a) D. Esrafilzadeh, J. M. Razal, S. E. Moulton, E. M. Stewart, G. G. Wallace, J. Control. Release 2013, 169, 313;

[14] T. S. Anirudhan, A. M. Mohan, Int. J. Biol.Macromol. 2018, 110, 167.

[15] J. Hoqie, B. Bhattacharjee, R. G. Prakash, K. Paramandham, J. Haldar, Biomacromolecules 2018, 19, 267.

[16] a) M. A. Xaplanteri, A. Andreou, G. P. Dinos, D. L. Kalpaxis, Nucleic Acids Res. 2003, 31, 5074; b) N. Polacek, M. J. Gomez, K. Ito, L. Xiong, Y. Nakamura, A. Mankin, Mol. Cell 2003, 11, 103. 


\section{WILEY-VCH}

[17] a) M. J. Donahue, A. Sanchez-Sanchez, S. J. Qu, R. M. Owens, Mater. Sci. Eng. $R$ Reports 2020, 140, 100546; b) L. Groenendaal, G. Zotti, P. H. Aubert, S. M. Waybright, J. R.

Reynolds, Adv. Mater. 2003, 15, 855; c) D. Aradilla, F. Estrany, C. Alemán, J. Phys. Chem. C 2011, 115, 8430; d) S. Kirchmeyer, K. Reuter, J. Mater. Chem. 2005, 15 2077; e) G. Fabregat,

B. Teixeira-Dias, L. J. del Valle, E. Armelin, F. Estrany, C. Alemán, ACS Appl. Mater. Interfaces 2014, 6, 11940.

[18] A. Puiggalí-Jou, P. Micheletti, F. Estrany, L. J. del Valle, C. Alemán, Adv. Healthc. Mater. 2017, 6, 1700453.

[19] A. Puiggalí-Jou, L. J. del Valle, C. Alemán, ACS Biomater. Sci. Eng. 2020, 6, 2135.

[20] M. Rivas, L. J. del Valle, A. M. Rodríguez-Rivero, P. Turon, J. Puiggalí, C. Alemán, ACS Biomater. Sci. Eng. 2018, 4, 3234.

[21] a) M. A. Khan, S. P. Armes, C. Perruchot, H. Ouamara, M. M. Chehimi, S. J. Greaves, J. F. Watts, Langmuir 2000, 16, 4171; b) G. Fabregat, B. Teixeira-Dias, L. J. del Valle, E. Armelin, F. Estrany, C. Alemán, ACS Appl. Mater. Interfaces 2014, 6, 11940.

[22] a) G. Zotti, S. Zecchin, G. Schiavon, F. Louwet, L. Groenendaal, X. Crispin, W. Osikowicz, W. Salaneck, M. Fahlman, Macromolecules 2003, 36, 3337; b) D. Aradilla, D. Azambuja, F. Estrany, J. I. Iribarren, C. A. Ferreira, C. Alemán, Polym. Chem. 2011, 2, 2548. [23] S. R. Taffarel, J. Rubio, Miner. Eng. 2010, 23, 771.

[24] X. Cao, Y. Huang, C. Tang, J. Wang, D. Jonson, Y. Fang, J. Environ. Sci. 2020, 88, 81.

[25] N. Sakmeche, S. Aeiyach, J. J. Aaron, M. Jouini, J. C. Lacroix, P. C. Lacaze, Langmuir 1999, 15, 2566.

[26] L. Preem, F. Bock, M. Hinnu, M. Putrin, K. Sagor, T. Tenson, A. Meos, J. Ostergaard, K. Kogermann, Pharmaceutics 2019, 11, 487.

[27] X. Xie, H. Wei, L. Kou, L. Ren, J. Zhou, Mater. Sci. Eng. Technol. 2020, 51, 850.

[28] M. Rivas, M. Pelechà, L. Franco, P. Turon, C. Alemán, L. J. Del Valle, J. Puiggalí, Int. J. Mol. Sci. 2019, 20, 5056. 


\section{WILEY-VCH}

[29] a) S. Chuanuwatanakul, O. Chailapakul, S. Motomizu, Anal. Sci. 2008, 24, 493; b) N.

Sebastian, W. C. Yu, D. Balram, Inorg. Chem. Front. 2019, 6, 82; c) S. Pilehvar, F. Dardenne, R. Blust, K. De Wael, Int. J. Electrochem. Sci. 2012, 7, 5000.

[30] M.-T. Hsieh, T.-J. Whang, J. Electroanal. Chem. 2017, 795, 130.

[31] Y. Qiao, C. M. Li, S. J. Bao, Z. Lu, Y. Hong, Chem. Commun. 2008, 1290.

[32] Y.-X. Huang, X.-W. Liu, J.-F. Xie, G.-P. Sheng, G.-Y. Wang, Y.-Y. Zhang, A. W. Xu, H.-Q. Yu, Chem. Commun. 2011, 47, 5795. 
Table 1. Percentage atomic composition (C 1s, N 1s, O 1s, S 2p and Cl 2p; in \%), S 2p / N 1s ratio $^{\mathrm{a}}$ and $\mathrm{S} 2 \mathrm{p} / \mathrm{Cl} 2 \mathrm{p}$ ratio $^{\mathrm{a}}$ obtained by XPS for PEDOT and PEDOT/CAM NPs.

\begin{tabular}{|c|c|c|c|c|c|c|c|}
\hline & \multicolumn{5}{|c|}{ Atomic composition (\%) } & \multirow[b]{2}{*}{$\mathbf{S} 2 \mathrm{p} / \mathrm{N} 1 \mathrm{~s}$} & \multirow[b]{2}{*}{ S 2p / Cl 2] } \\
\hline & C 1s & N 1s & O 1s & S 2p & Cl 2p & & \\
\hline PEDOT & 49.00 & - & 47.29 & 3.71 & - & - & - \\
\hline PEDOT/CAM & 57.20 & 0.55 & 40.28 & 1.14 & 0.83 & 4.14 & 2.75 \\
\hline
\end{tabular}

${ }^{\mathrm{a}}$ Each CAM molecule contains two nitrogen and two chlorine atoms (see Scheme 1).

\section{Monomer: EDOT Drug: Chloramphenicol}

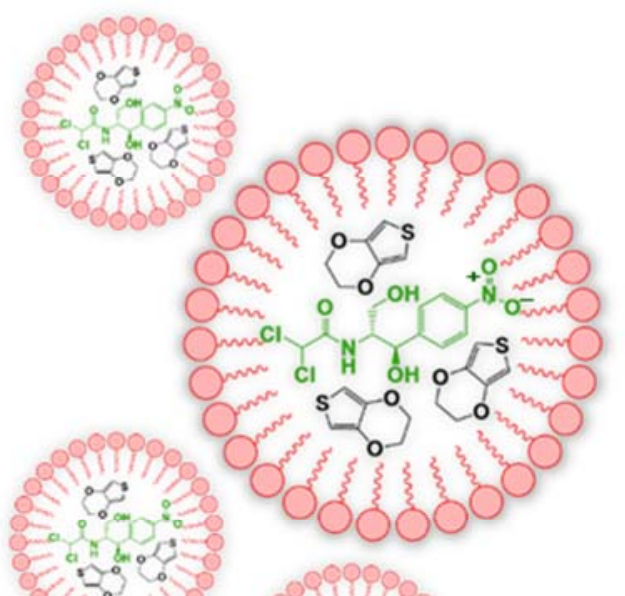

Oxidizing agent: Ammonium persulfate (APS)
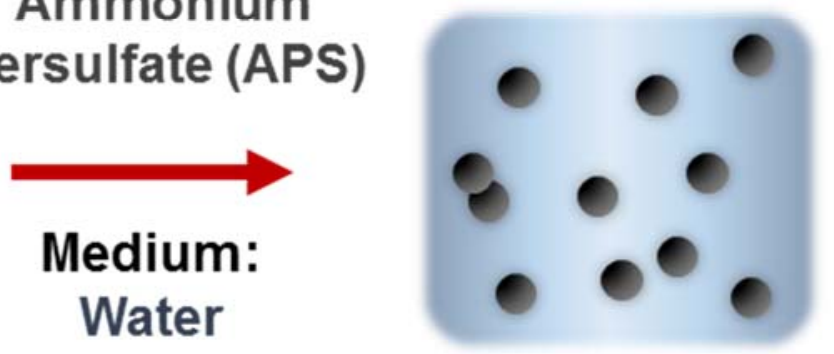

PEDOT/CAM NPS

\section{Dopant \& surfactant: \\ Dodecyl benzen sulfonic acid (DBSA)}

Scheme 1. Synthesis of PEDOT and PEDOT/CAM NPs 
(a)

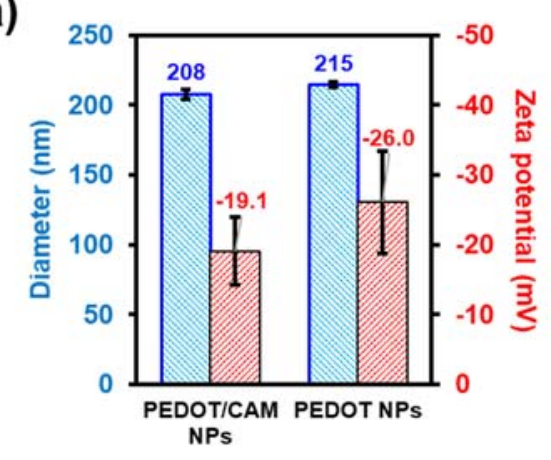

(c)

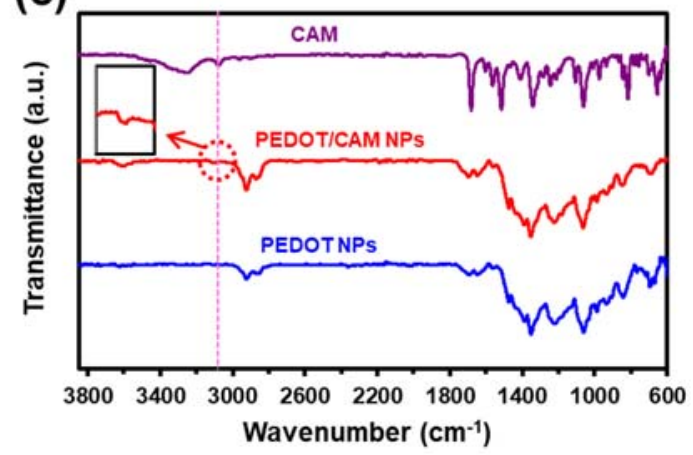

(b)

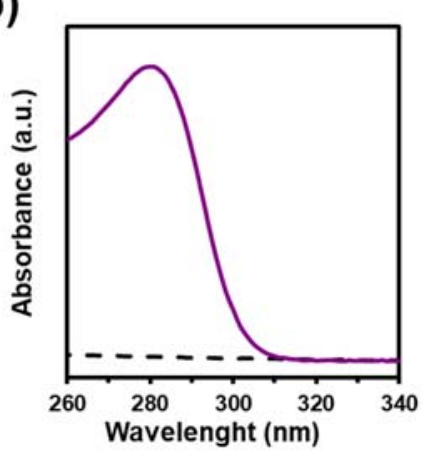

(d)

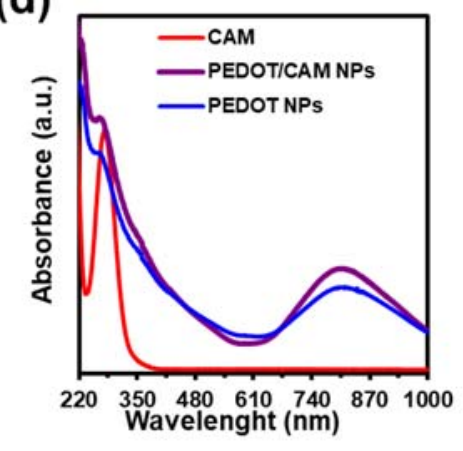

Figure 1. (a) Diameter and $\zeta$-potential of PEDOT and PEDOT/CAM NPs $(n=30)$. (b) UVVis Spectra of CAM released from PEDOT/CAM in ethanol (solid line) compared to the blank (dashed line). (c) FTIR spectra of free CAM, PEDOT and PEDOT/CAM NPs.
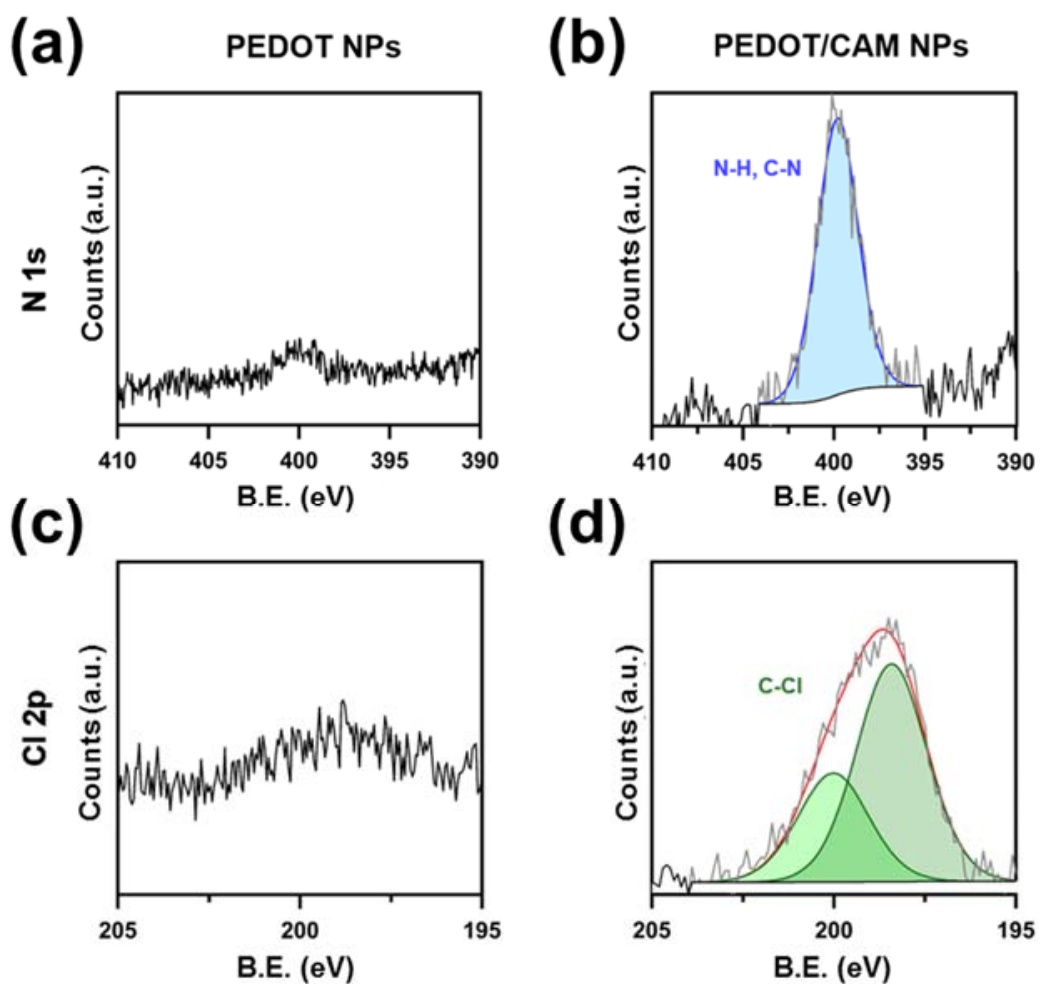

Figure 2 High-resolution XPS spectra of ( $a, b) \mathrm{N}$ 1s and (c, d) $\mathrm{Cl} 2 \mathrm{p}$ regions for PEDOT and (b, d) PEDOT/CAM NPs. 


\section{WILEY-VCH}

(a)
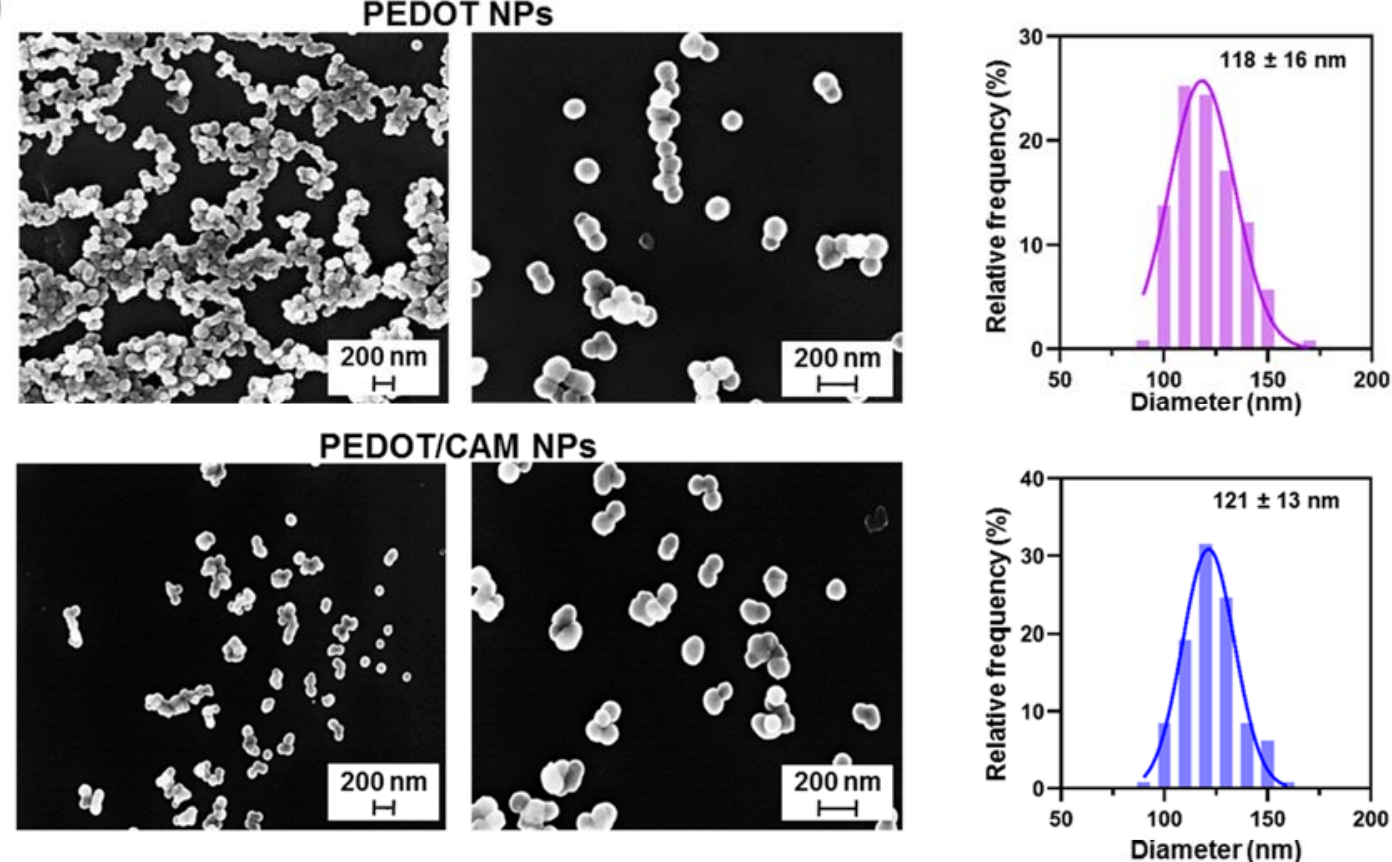

(b)
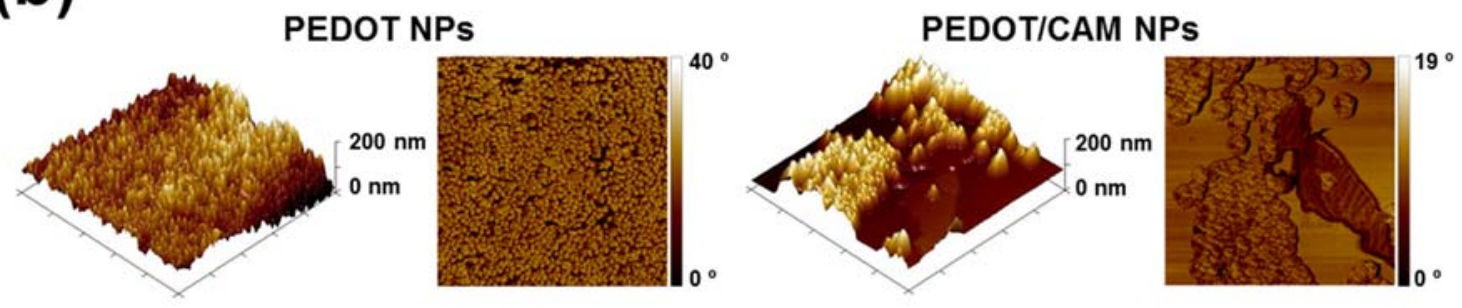

Figure 3. For PEDOT and PEDOT/CAM NPs: (a) SEM micrographs (left, 50k $\times$ magnification; right, $100 \mathrm{k} \times$ magnification) and diameter distribution histogram $(n=250)$; and (b) 3D topographic (left) and 2D phase (right) AFM images.

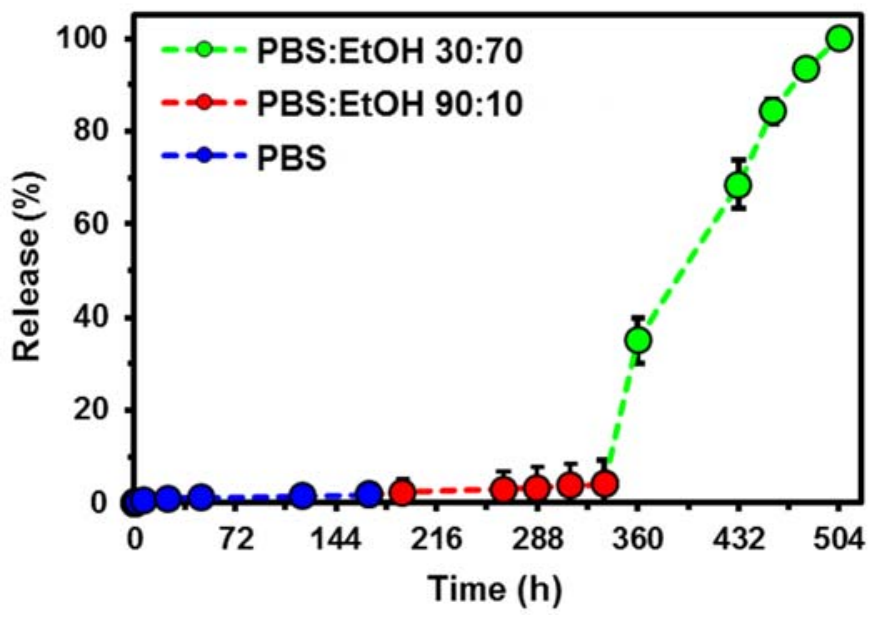

Figure 4. Drug release from PEDOT/CAM NPs in PBS (first week), PBS:EtOH 90:10 (second week) and PBS:EtOH 30:70 (third week) at $37^{\circ} \mathrm{C}(n=3)$. 
WILEY-VCH

(a)

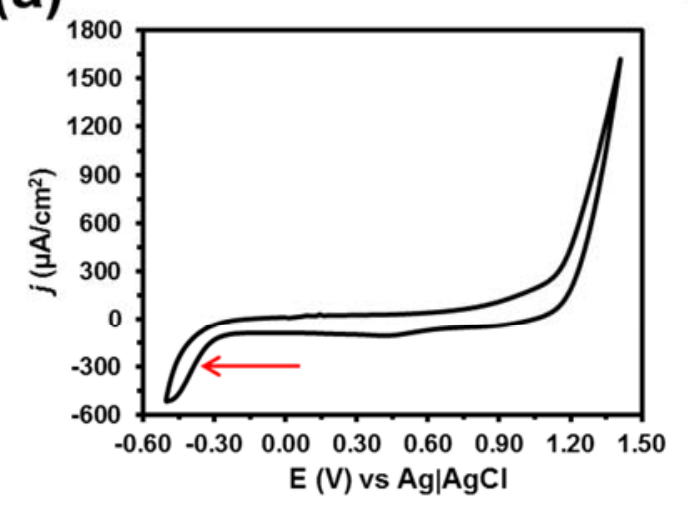

(c)

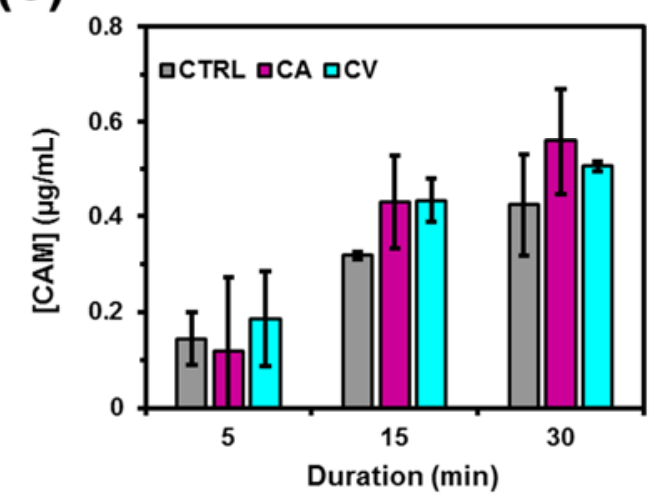

(b)

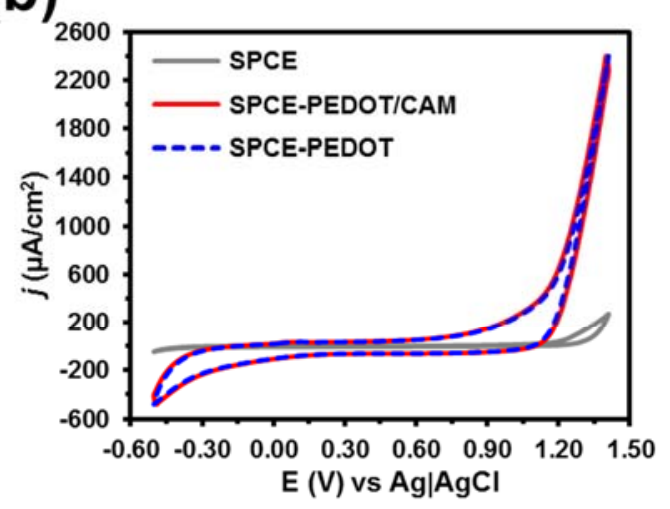

(d)

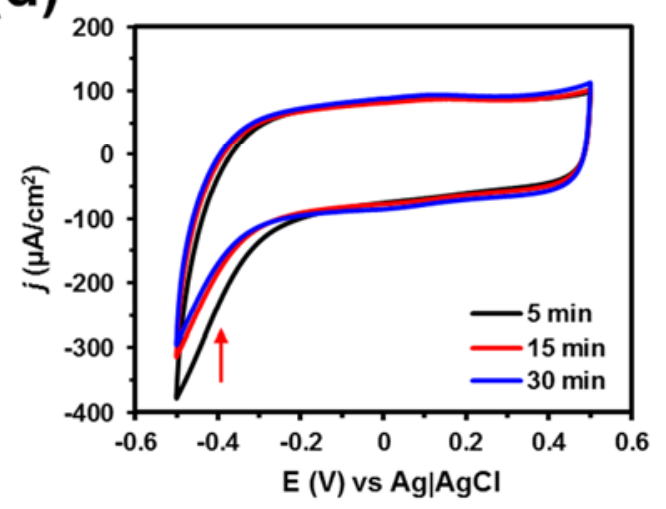

Figure 5. Cyclic voltammograms recorded from -0.50 to $1.40 \mathrm{~V}$ (scan rate: $100 \mathrm{mV} / \mathrm{s}$ ) for (a) free CAM and (b) comparison of the bare electrode (SPCE) and the coated electrodes (SPCEPEDOT and SPCE-PEDOT/CAM). (c) CAM release during 5, 15 and $30 \mathrm{~min}(n=3)$ without electrical stimulation (CTRL) and with two different types of electrical stimulation CA (1.00 $\mathrm{V}$ ) and CV (from -0.50 to $0.50 \mathrm{~V}$ ). (d) Cyclic voltammograms recorded from -0.50 to $0.50 \mathrm{~V}$ (scan rate: $100 \mathrm{mV} / \mathrm{s}$ ) for SPCE-PEDOT/CAM after 5, 15 and $30 \mathrm{~min}$ of CV stimulation.

(a)

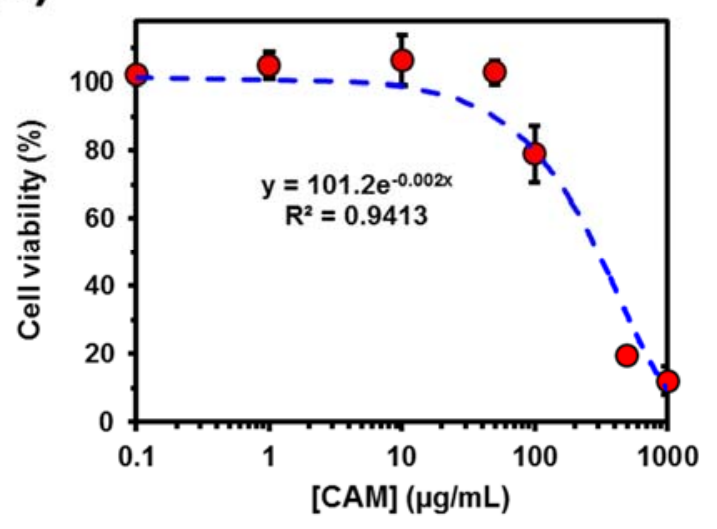

(b)

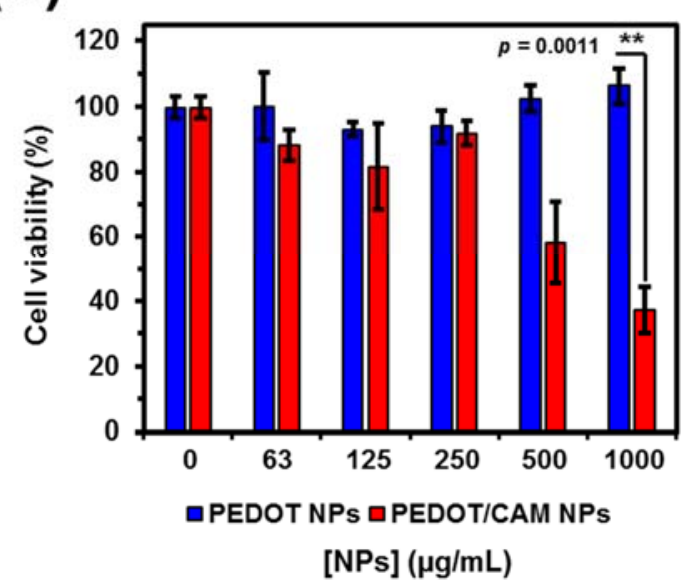

Figure 6. Dose-dependent viability of MG-63 cells treated with (a) free CAM and (b) PEDOT vs PEDOT/CAM NPs $(n=3)$. Statistical comparison of values was based on a 2-way ANOVA using Tukey's test for pair-wise comparison with $\mathrm{p}<0.05$. 
(a)

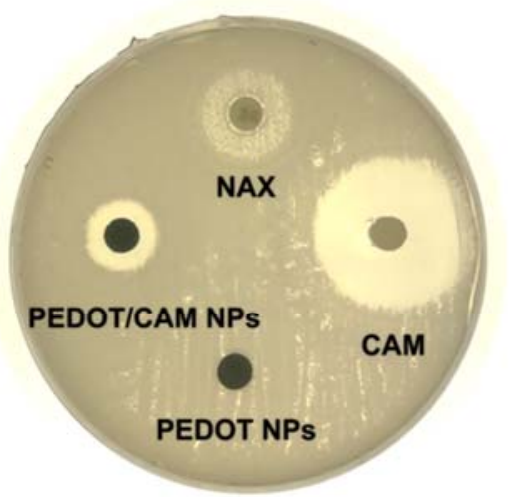

(c)

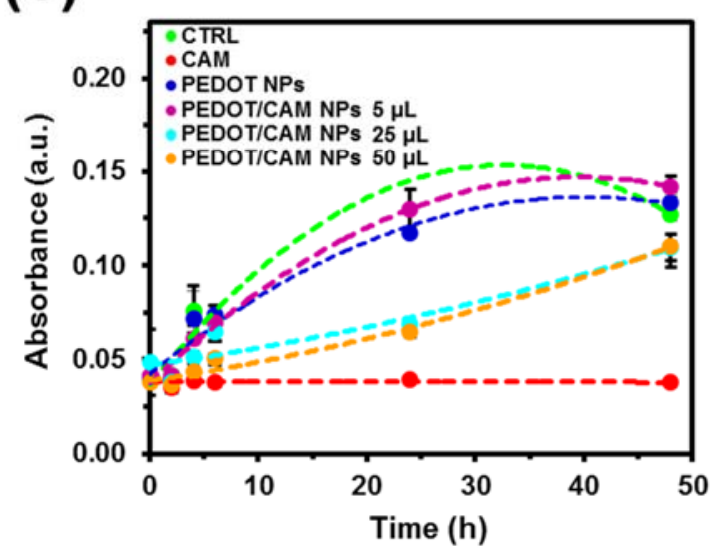

(b)

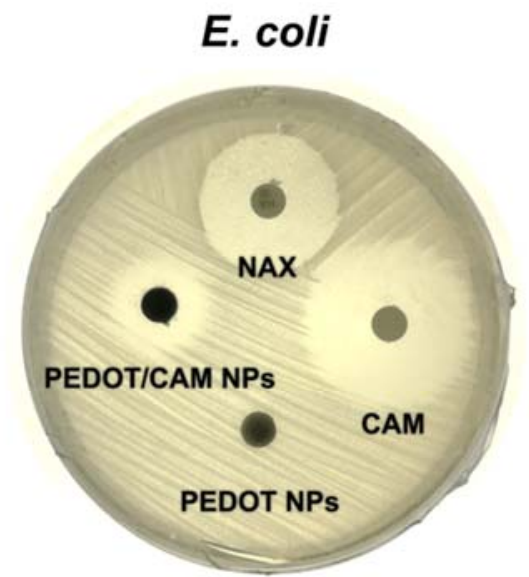

(d)

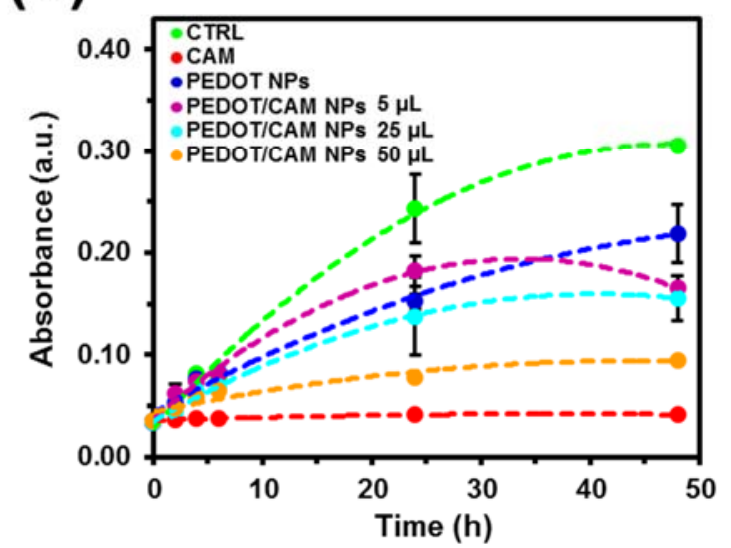

Figure 7. Bactericidal activity of free CAM, free NAX, PEDOT NPs and PEDOT/CAM NPs against (a, c) S. sanguinis and (b, d) E. coli bacteria $(n=3)$ : $(a, b)$ Inhibition halos observed using the disk diffusion method; and (c, d) growth curves obtained by treating the bacteria cultures with LB medium (CTRL), free CAM, PEDOT NPs and different volumes of PEDOT/CAM NPs $(5 \mu \mathrm{L}, 25 \mu \mathrm{L}$ and $50 \mu \mathrm{L})$. The error bar is not visible when the standard deviation is smaller than the size of the solid circle used to represent the values. 


\section{WILEY-VCH}

(a)

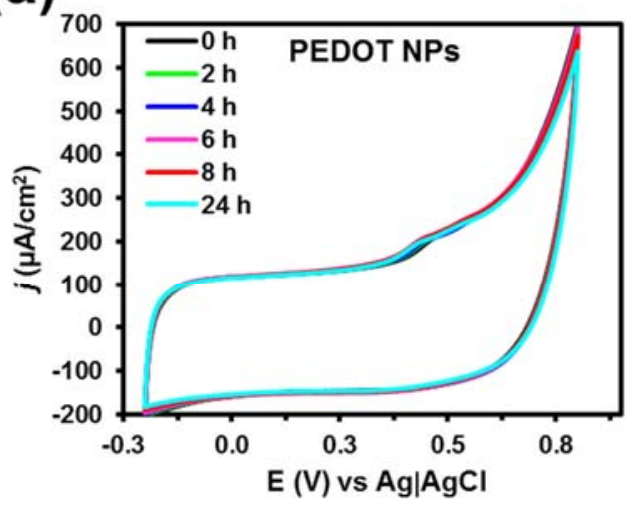

(b)

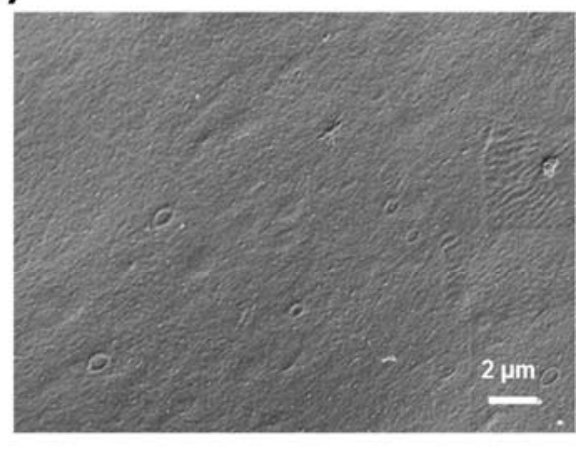

(c)

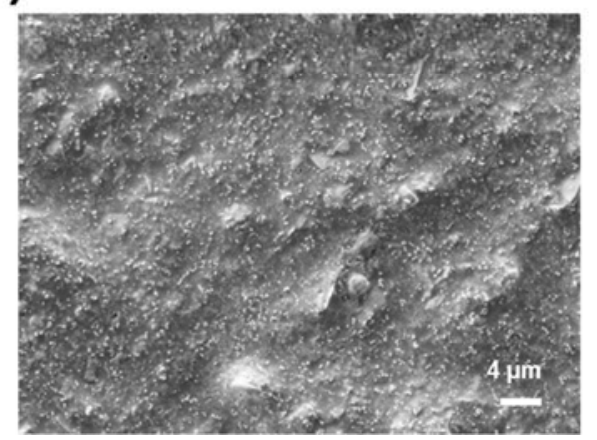

Figure 8. (a) Cyclic voltammograms of PEDOT NPs, which are coating SPCEs, in the culture medium ( $\mathrm{NaHCO}_{3}$-supplemented DMEM) recorded at different time intervals $(0,2,4,6,8$ and $24 \mathrm{~h}$ ). (b) Representative SEM micrograph of the SPCE coated with PEDOT NPs after 0 $\mathrm{h}$ and $24 \mathrm{~h}$ in culture medium. 


\section{WILEY-VCH}

(a)

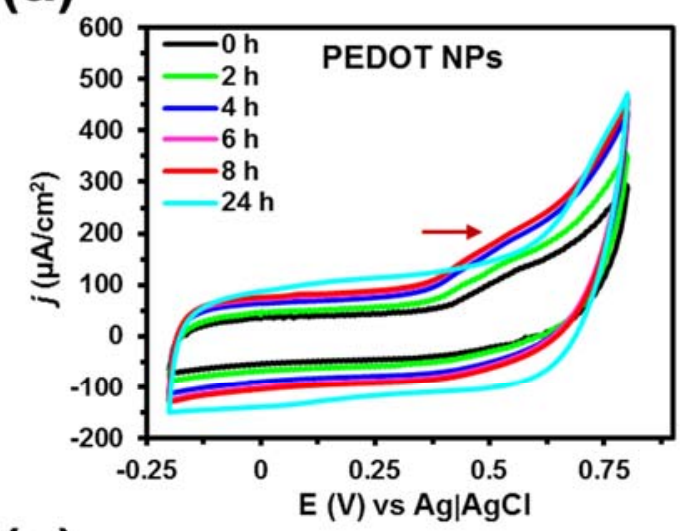

(c)

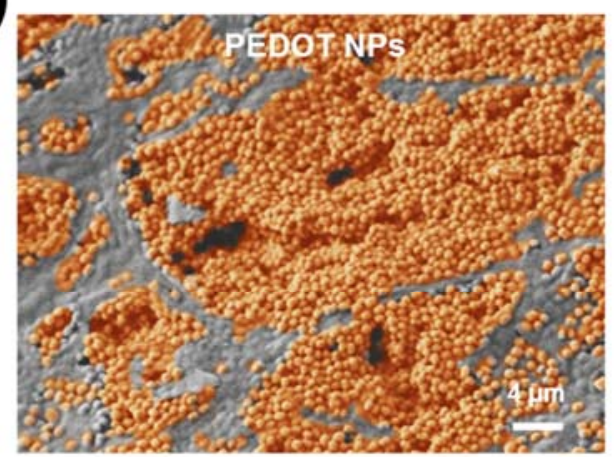

(e)

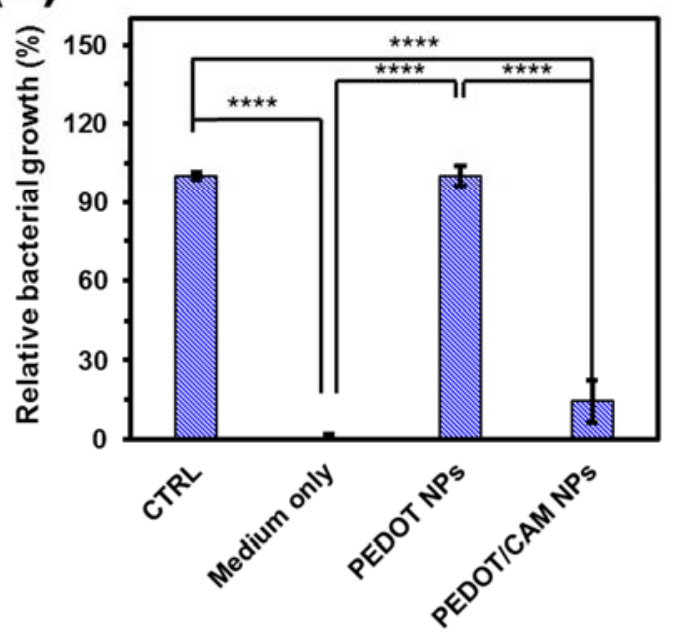

(b)

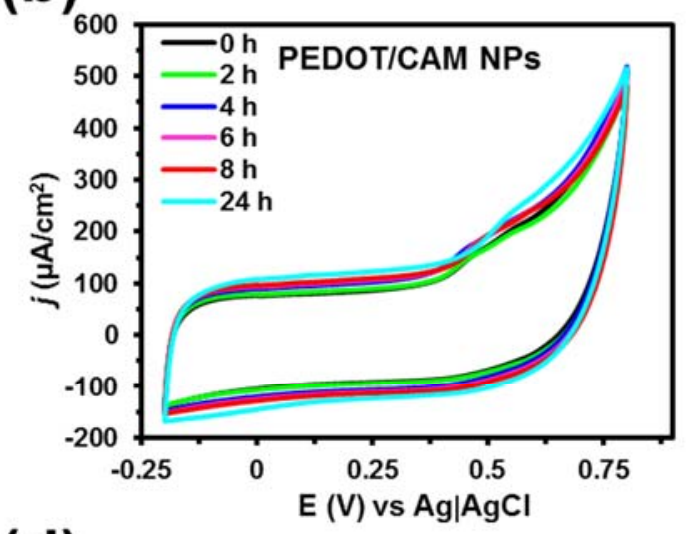

(d)

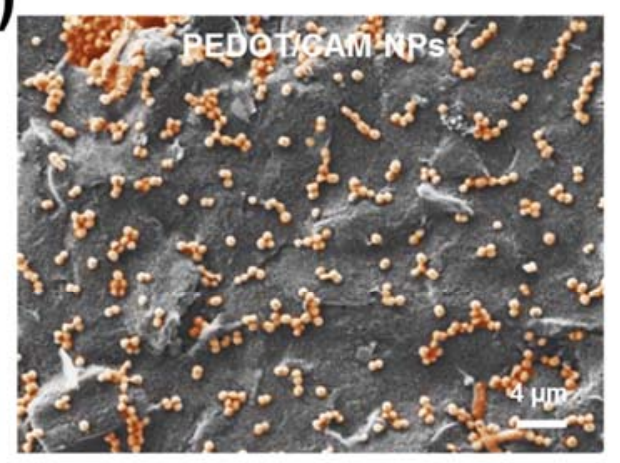

(f)

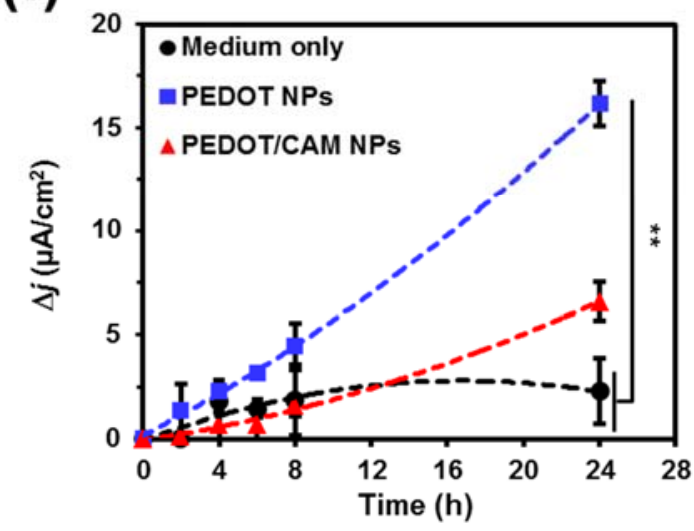

Figure 9. Cyclic voltammograms for SPCEs coated with (a) PEDOT and (b) PEDOT/CAM NPs recorded at different incubation times $(0,2,4,6,8$ and $24 \mathrm{~h})$ in the culture medium $\left(\mathrm{NaHCO}_{3}\right.$-supplemented DMEM) with $S$. sanguinis. Initial and final potentials: $-0.20 \mathrm{~V}$; reversal potential: $0.80 \mathrm{~V}$; and scan rate: $100 \mathrm{mV} / \mathrm{s}$. SEM micrograph of the surface of electrodes coated with (c) PEDOT and (d) PEDOT/CAM NPs after $24 \mathrm{~h}$ in presence of $S$. sanguinis (artificially coloured in orange). (e) Relative bacterial growth calculated through the variation of the absorbance at $600 \mathrm{~nm}$ after $24 \mathrm{~h}$ of culture $(n=3)$. (f) Variation in peak current density with the incubation time relative to $0 \mathrm{~h}$ for the voltammograms displayed in (a) and (b) $(n=3)$. The statistical comparison of values in (e) and (f) was based on one-way ANOVA with multi-comparison test, with $\mathrm{p}<0.0001(* * * *), \mathrm{p}<0.001(* * *)$, and $\mathrm{p}<0.01$ $(* *)$. 


\section{WILEY-VCH}

(a)

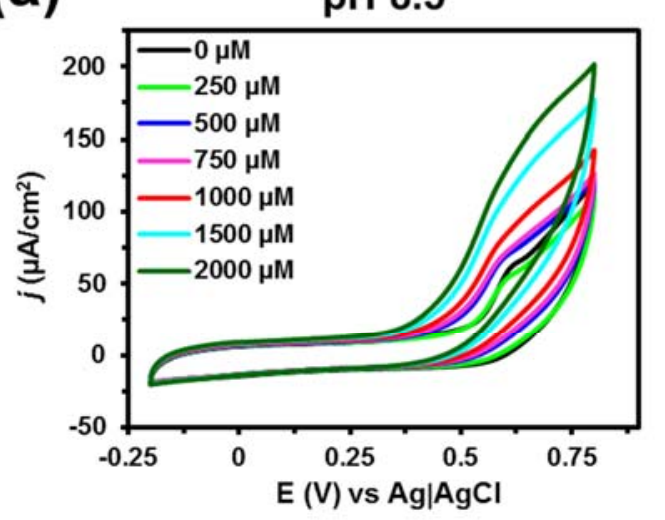

(b)

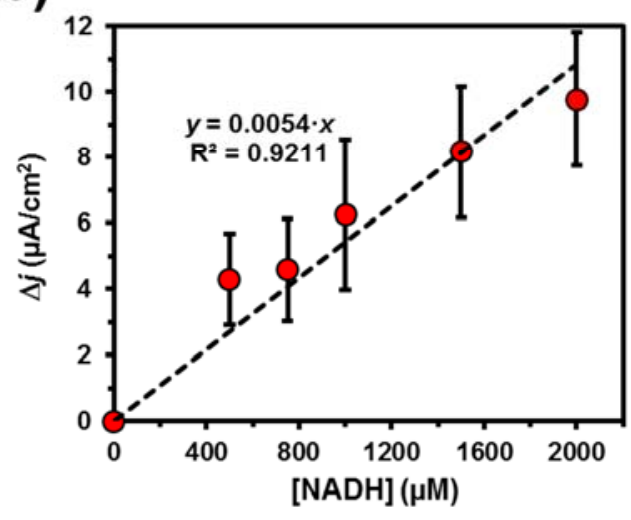

(c)

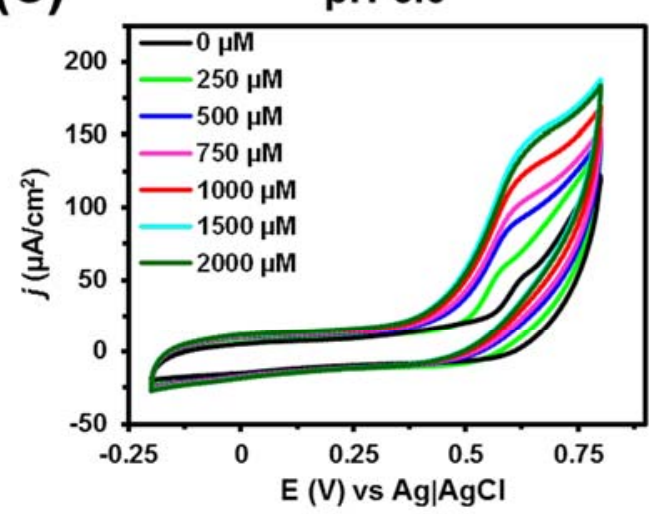

(d)

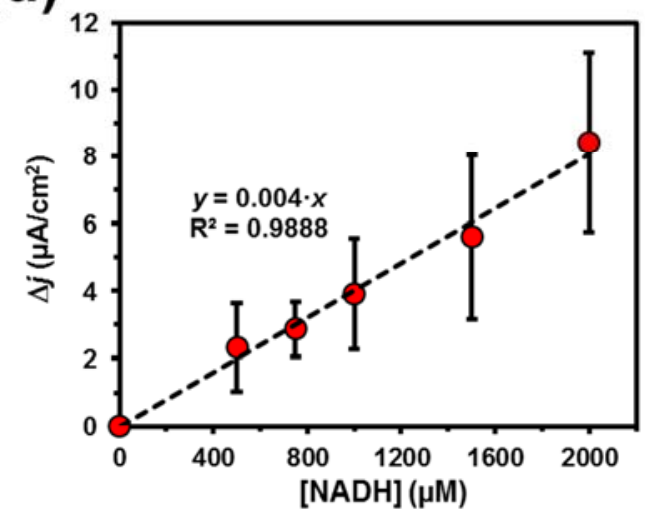

Figure 10. (a, c) Cyclic voltammograms and (b, d) calibration curves for SPCE coated with PEDOT NPs using cell culture medium at (a) $\mathrm{pH} 8.5$ and (b) $\mathrm{pH} 6.0$ with different NADH concentrations $(n=3)$. Voltammograms were recorded using the following operational conditions: Initial and final potentials: $-0.20 \mathrm{~V}$; reversal potential: $+0.80 \mathrm{~V}$; and scan rate: 100 $\mathrm{mV} / \mathrm{s}$. Calibration curves are expressed as the relative variation of the current density against the NADH concentrations (data taken from voltammograms displayed in (a) and (c)). 


\section{WILEY-VCH}

A highly sensitive electrode consisting of antibiotic-loaded conducting polymer nanoparticles has been developed to monitor the inhibition of bacterial growth in real time. The engineered a nanotheranostic system, which able to sense the infection progression while releasing the antibiotic, has been developed using of chloramphenicol, a wide-spectrum antibiotic, and electroactive poly(3,4-ethylendioxythiophene) nanoparticles.

H. Enshaei, A. Puiggalí-Jou, * L. J. del Valle, P. Turon, N. Saperas* Carlos Alemán*

\section{Nanotheranostic Interface Based on Antibiotic-Loaded Conducting Polymer Nanoparticles for Real-Time Monitoring of Bacterial Growth Inhibition}

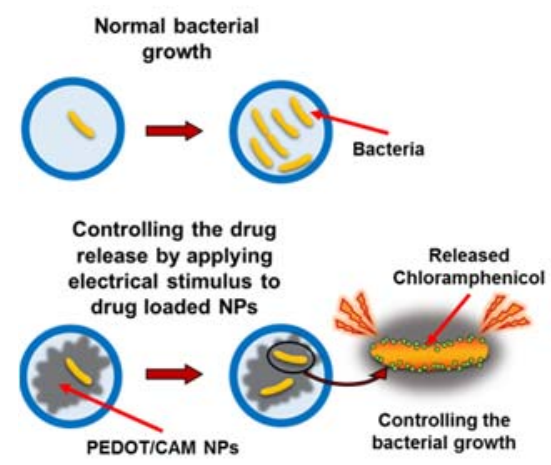


Copyright WILEY-VCH Verlag GmbH \& Co. KGaA, 69469 Weinheim, Germany, 2018.

\section{Supporting Information}

\section{Nanotheranostic Interface Based on Antibiotic-Loaded Conducting Polymer Nanoparticles for Real-Time Monitoring of Bacterial Growth Inhibition}

Hamidreza Enshaei, Anna Puiggalí-Jou, * Luis J. del Valle, Pau Turon, Núria Saperas* and Carlos Alemán*

\section{METHODS}

Synthesis of poly(3,4-ethylenedioxythiophene) nanoparticles (PEDOT NPs). A $30 \mathrm{~mL}$ Corex tube was filled with $15.8 \mathrm{~mL}$ of milli-Q water. After this, $96 \mu \mathrm{L}$ of dodecyl benzenesulfonic acid (DBSA) were added and the solution was stirred at $40{ }^{\circ} \mathrm{C}$ for $1 \mathrm{~h}$ with a magnetic stirrer set at $750 \mathrm{rpm}$ and protected from light with aluminum foil. $72 \mu \mathrm{L}$ of $3,4-$

ethylenedioxythiophene (EDOT) monomer and $2 \mathrm{~mL}$ of ethanol were then added slowly, and the mixture was allowed to stir for $1 \mathrm{~h}$ at $750 \mathrm{rpm}$ and $40{ }^{\circ} \mathrm{C}$. Finally, $0.73 \mathrm{mg}$ of ammonium persulfate (APS) dissolved in $2 \mathrm{~mL}$ of milli-Q water were added to the mixture drop by drop while stirring. The reaction was maintained in agitation at $40{ }^{\circ} \mathrm{C}$ overnight. In this process, the color of the reaction mixture changed from light grey to dark blue. No sedimentation was observed after the reaction occurred, indicating a good colloidal stability. The side products and unreacted chemicals were removed by a sequence of 3 centrifugations at $11000 \mathrm{rpm}$ for 40 min at $4{ }^{\circ} \mathrm{C}$. After each centrifugation, the resulting supernatants were decanted and the pellet was dispersed in $15 \mathrm{~mL}$ of deionized water by using a vortex and a sonication bath (15 min at room temperature). The last pellet was left under vacuum in the same tube for two days, then weighted (33 mg) and dispersed in the corresponding media at the desired concentration.

Synthesis of chloramphenicol-loaded PEDOT NPs (PEDOT/CAM NPs). $96 \mu \mathrm{L}$ of DBSA were added to a $30 \mathrm{~mL}$ tube filled with $15.8 \mathrm{~mL}$ of milli-Q water and the solution was stirred for 1 


\section{WILEY-VCH}

$\mathrm{h}$ at $750 \mathrm{rpm}$ and $40{ }^{\circ} \mathrm{C}$. After this, $72 \mu \mathrm{L}$ of EDOT and $2 \mathrm{~mL}$ of drug solution $(8 \mathrm{mg} / \mathrm{mL}$ CAM in ethanol) were added drop by drop while stirring and the resulting solution was stirred at $750 \mathrm{rpm}$ and $40^{\circ} \mathrm{C}$ during $1 \mathrm{~h}$. Finally, $0.73 \mathrm{mg}$ of APS were dissolved in $2 \mathrm{~mL}$ of milli-Q water and were added to the mixture. The reaction was protected from light (aluminum foil) and maintained in agitation at $40{ }^{\circ} \mathrm{C}$ overnight. The color of the reaction mixture changed from light grey to dark blue. No sedimentation was observed after the reaction occurred, indicating good colloidal stability. The side products, extra drug and unreacted chemicals were removed by a sequence of 3 centrifugations at $11000 \mathrm{rpm}$ for $40 \mathrm{~min}$ at $4{ }^{\circ} \mathrm{C}$. The resulting supernatants were decanted and the pellet was re-dispersed in deionized water by using a vortex and an ultrasonic bath (15 min at room temperature). The last pellet was left under vacuum for two days, then weighted and re-dispersed in the corresponding media at the desired concentration.

Determination of the drug loading ratio. The drug content was determined by taking $10 \mu \mathrm{L}$ of PEDOT/CAM NPs suspension (10 mg/mL NPs in milli-Q water) into $990 \mu \mathrm{L}$ of drug solvent (ethanol). The suspension was sonicated and vortexed for $10 \mathrm{~min}$, leading to a complete drug release in the alcoholic medium. Then, the NPs dispersion was centrifuged with a microcentrifuge for $15 \mathrm{~min}$ at $2500 \mathrm{rpm}$. Finally, the supernatant was evaluated using a UV-Vis spectrometer. The calibration curve was prepared with the drug dissolved in ethanol and read at $280 \mathrm{~nm}$ (Figure S3). The same procedure was applied to determine the drug released during the dialysis or after the electrical stimuli assays (see below).

The loading capacity (LC, in \%) was calculated using the following equation:

$$
L C=\frac{\left(\mathrm{W}_{i}-W_{f}\right)}{W_{N P s}} \times 100
$$

where $W_{i}$ indicates CAM initial mass, $W_{f}$ CAM final mass, and $W_{N P s}$ total NPs mass. 


\section{WILEY-VCH}

Dynamic Light Scattering (DLS). DLS studies were performed using NanoBrook Omni Zeta Potential Analyzer from Brookheaven Instruments. Measurement consisted of 3 runs of $120 \mathrm{~s}$ each, which were averaged to obtain the effective diameter (Deff). Samples were analyzed at $25^{\circ} \mathrm{C}$ using a scattering angle of $90^{\circ}$. In order to know the zeta $(\zeta)$-potential, particles were resuspended in $1 \mathrm{mM} \mathrm{KCl}$ solution and 30 consecutive measurements were taken of each sample.

Fourier transform infrared (FTIR) Spectroscopy. FTIR transmittance spectra were recorded on a FTIR Jasco 4100 spectrophotometer. Samples were deposited on an attenuated total reflection accessory (Top-plate) with a diamond crystal (Specac model MKII Golden Gate Heated Single Reflection Diamond ATR). For each sample, 64 scans were performed between 4000 and $600 \mathrm{~cm}^{-1}$ with a resolution of $4 \mathrm{~cm}^{-1}$.

RAMAN spectroscopy. Samples were characterized by micro-Raman spectroscopy using a commercial Renishaw inVia Qontor confocal Raman microscope. The Raman setup consisted of a laser (at $785 \mathrm{~nm}$ with a nominal $300 \mathrm{~mW}$ output power) directed through a microscope (specially adapted Leica DM2700 M microscope) to the sample, after which the scattered light is collected and directed to a spectrometer with a 1200 lines $\cdot \mathrm{mm}^{-1}$ grating. The exposure time was $10 \mathrm{~s}$, the laser power was adjusted to $0.001-0.05 \%$ of its nominal output power depending on the sample, and each spectrum was collected with 30 accumulations.

X-ray photoelectron spectroscopy (XPS). XPS analyses were performed in a SPECS system equipped with a high-intensity twin-anode X-ray source XR50 of Mg/Al (1253 eV/1487 eV) operating at $150 \mathrm{~W}$, placed perpendicular to the analyzer axis, and using a Phoibos $150 \mathrm{MCD}$ $9 \mathrm{XP}$ detector. The X-ray spot size was $650 \mu \mathrm{m}$. The pass energy was set to 25 and $0.1 \mathrm{eV}$ for the survey and the narrow scans, respectively. Charge compensation was achieved with a 


\section{WILEY-VCH}

combination of electron and argon ion flood guns. The energy and emission current of the electrons were $4 \mathrm{eV}$ and $0.35 \mathrm{~mA}$, respectively. For the argon gun, the energy and the emission current were $0 \mathrm{eV}$ and $0.1 \mathrm{~mA}$, respectively. The spectra were recorded with pass energy of $25 \mathrm{eV}$ in $0.1 \mathrm{eV}$ steps at a pressure below $6 \times 10^{-9} \mathrm{mbar}$. These standard conditions of charge compensation resulted in a negative but perfectly uniform static charge. The $\mathrm{C} 1 \mathrm{~s}$ peak was used as an internal reference with a binding energy of $284.8 \mathrm{eV}$. The surface composition was determined using the manufacturer's sensitivity factors.

Scanning electron microscopy (SEM). SEM micrographs were obtained using a Focused Ion Beam Zeiss Neon 40 scanning electron microscope operating at $5 \mathrm{kV}$. Samples were mounted on a double-side adhesive carbon disc and sputter-coated with a thin layer of carbon to prevent sample charging problems.

Atomic Force Microscopy (AFM). AFM was conducted to obtain topographic images of the NPs surface using silicon TAP $150-$ G probe (Budget Sensors, Bulgaria) with a frequency of $150 \mathrm{kHz}$ and a force constant of $5 \mathrm{~N} / \mathrm{m}$. Images were obtained with a Molecular Imaging PicoSPM microscope using a NanoScope IV controller under ambient conditions in tapping mode. AFM measurements were performed on various parts of the samples, which produced reproducible images similar to those displayed in this work.

Cytotoxicity Evaluation. The cytotoxicity of free CAM, PEDOT, and PEDOT/CAM NPs was evaluated by the MTT assay using the MG-63 cell line. Free CAM was dissolved in ethanol (the final concentration of ethanol in the cell media was smaller than $10 \%$ ). All the other substances were prepared in milli-Q water. Cells were seeded at a density of $20 \times 10^{4}$ cells per well $(100 \mu \mathrm{L}$ each) in 96-well plates and incubated overnight. Subsequently, the cells were exposed to a series of increasing free CAM, PEDOT, and PEDOT/CAM NPs concentrations. 


\section{WILEY-VCH}

CAM concentrations were $0.1,1,10,50,100,500$ and $1000 \mu \mathrm{g} / \mathrm{mL}$. PEDOT and

PEDOT/CAM NPs concentrations were $0.0655,0.125,0.25,0.5$ and $1 \mathrm{mg} / \mathrm{mL}$. Cells were incubated with the treatment for $24 \mathrm{~h}$. Next day, the percentage of viable cells relative to untreated control was determined on the basis of the mitochondrial conversion of 3-(4,5dimethylthiazol-2-yl)-2,5-diphenyltetrazolium bromide to formazan. The results were expressed as the mean value \pm standard deviation (SD). All the experiments were performed in triplicate. Statistical comparison of values was based on a 2-way ANOVA using Tukey's test for pair-wise comparison with $\mathrm{p}<0.05$.

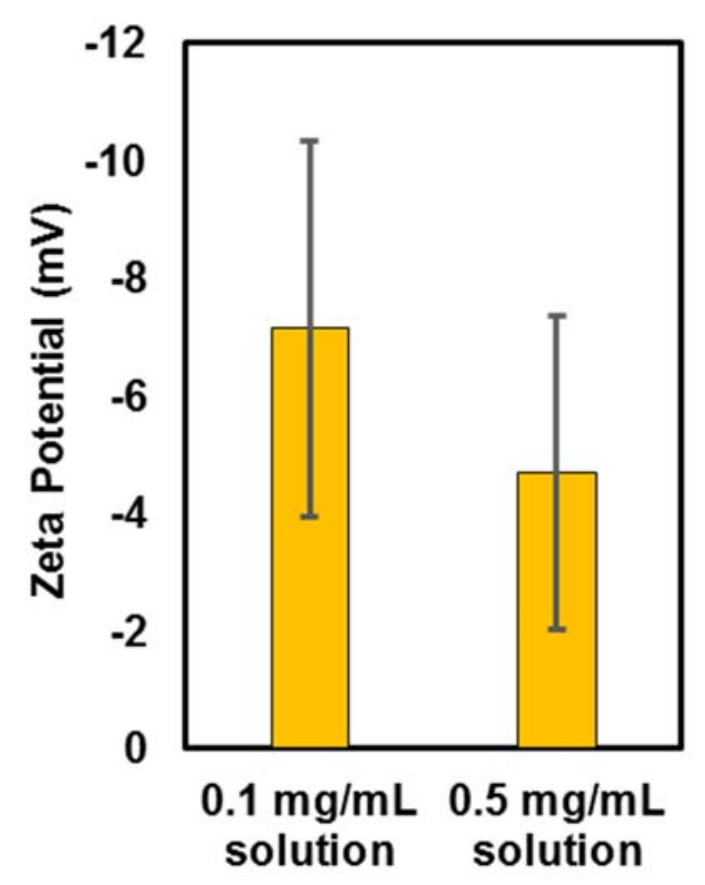

Figure S1. $\zeta$-potential of CAM solutions. 


\section{WILEY-VCH}

(a)

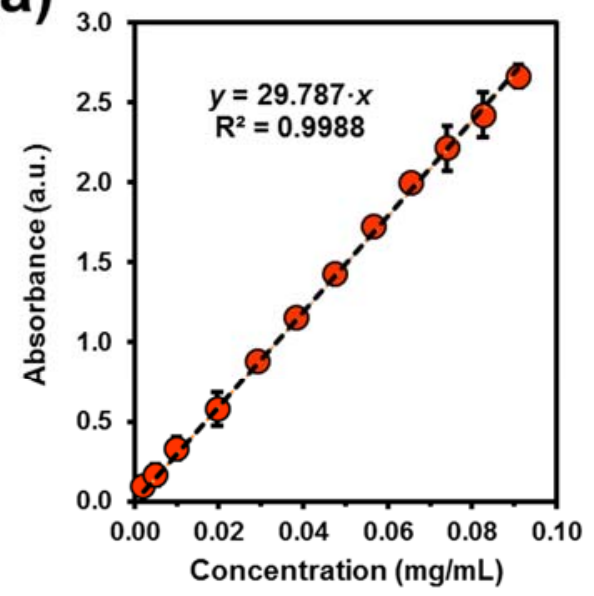

(b)

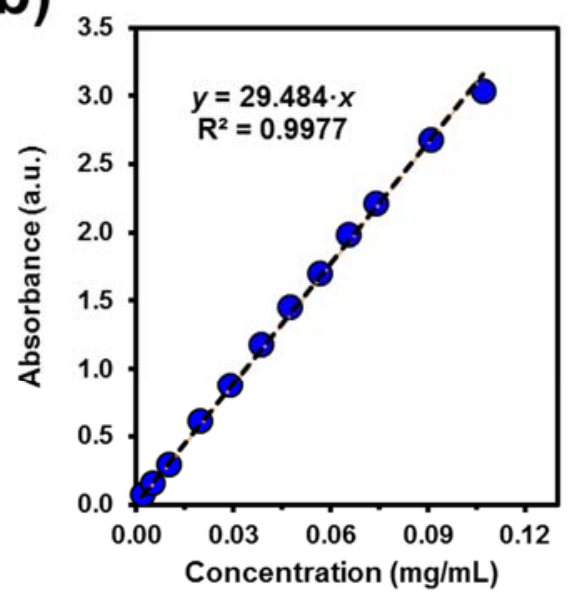

(c)

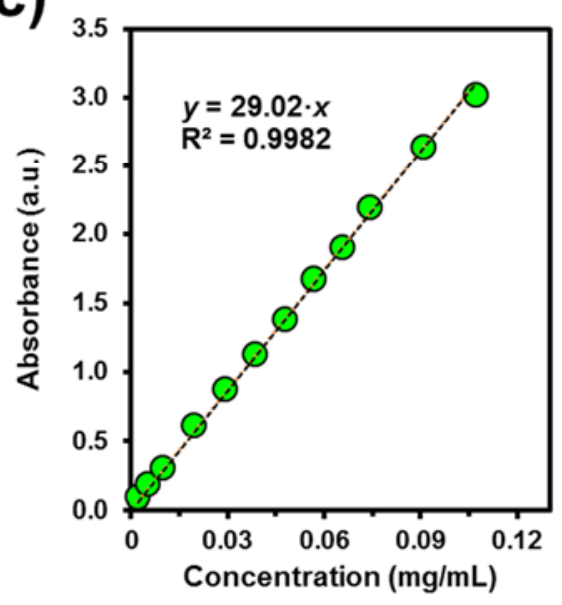

Figure S2. Calibration curves for CAM in (a) PBS, (b) PBS with $10 \%$ ethanol (PBS:EtOH 90:10), and (c) PBS with $70 \%$ ethanol (PBS:EtOH 30:70).

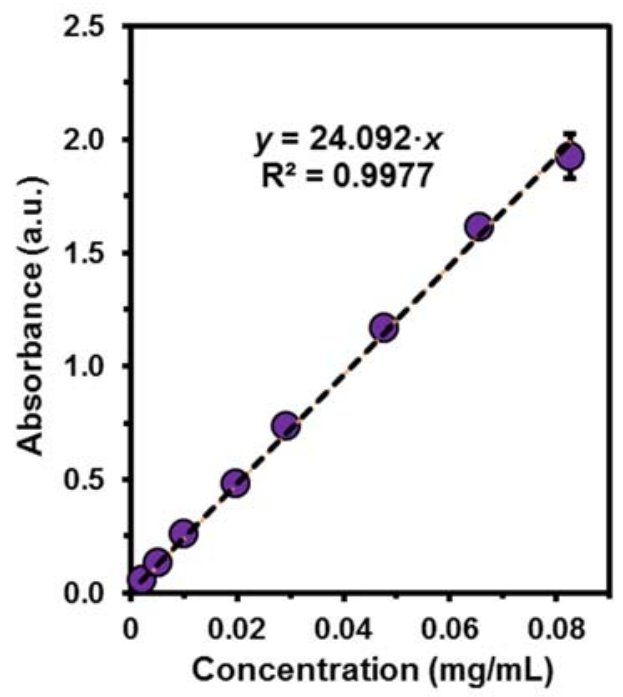

Figure S3. Calibration curve for CAM in ethanol. 


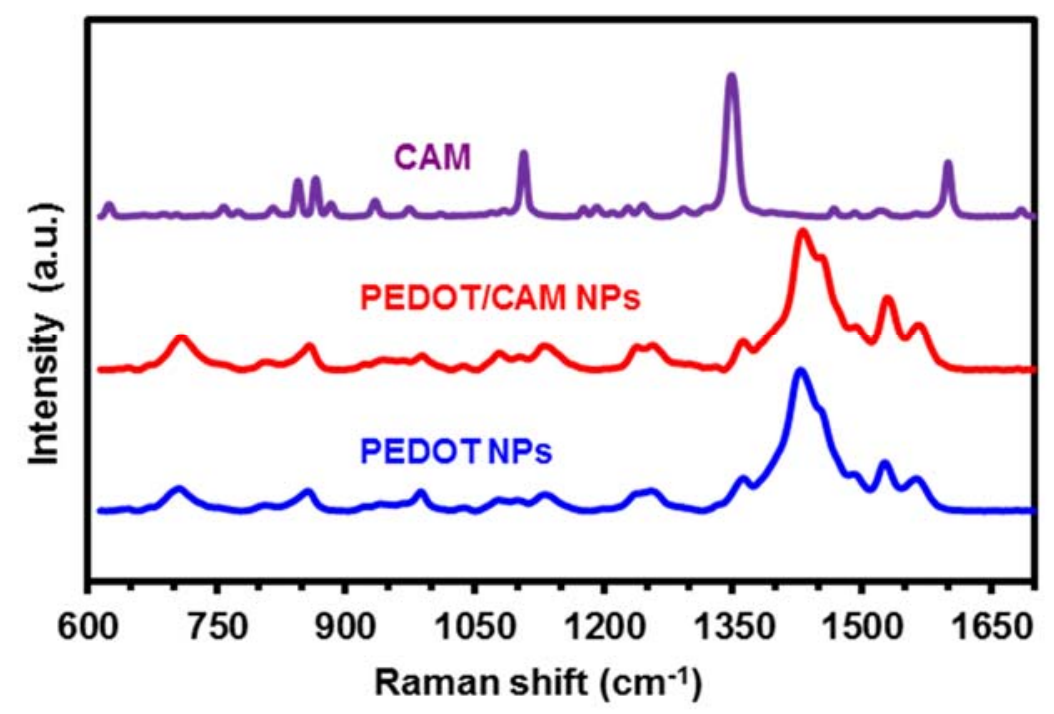

Figure S4. Raman spectra of free CAM, PEDOT and PEDOT/CAM NPs.

CAM main bands at 1107,13351 and $1598 \mathrm{~cm}^{-1}$ are attributed to the $\mathrm{C}-\mathrm{O}$ bending vibration, $\mathrm{C}-\mathrm{H}$ bond stretching vibration and $-\mathrm{NO}_{2}$ bending vibration, respectively. PEDOT NPs principal bands, which appear at 1436, 1231, 1376 and $1508 \mathrm{~cm}^{-1}$, correspond to the symmetric stretching mode of the aromatic $\mathrm{C}-\mathrm{C}$ bond and the antisymmetric stretching vibration of $\mathrm{C}_{\alpha}-\mathrm{C}_{\beta}$. Finally, the spectrum recorded for PEDOT/CAM NPs displays only the fingerprints of PEDOT NPs. 
WILEY-VCH
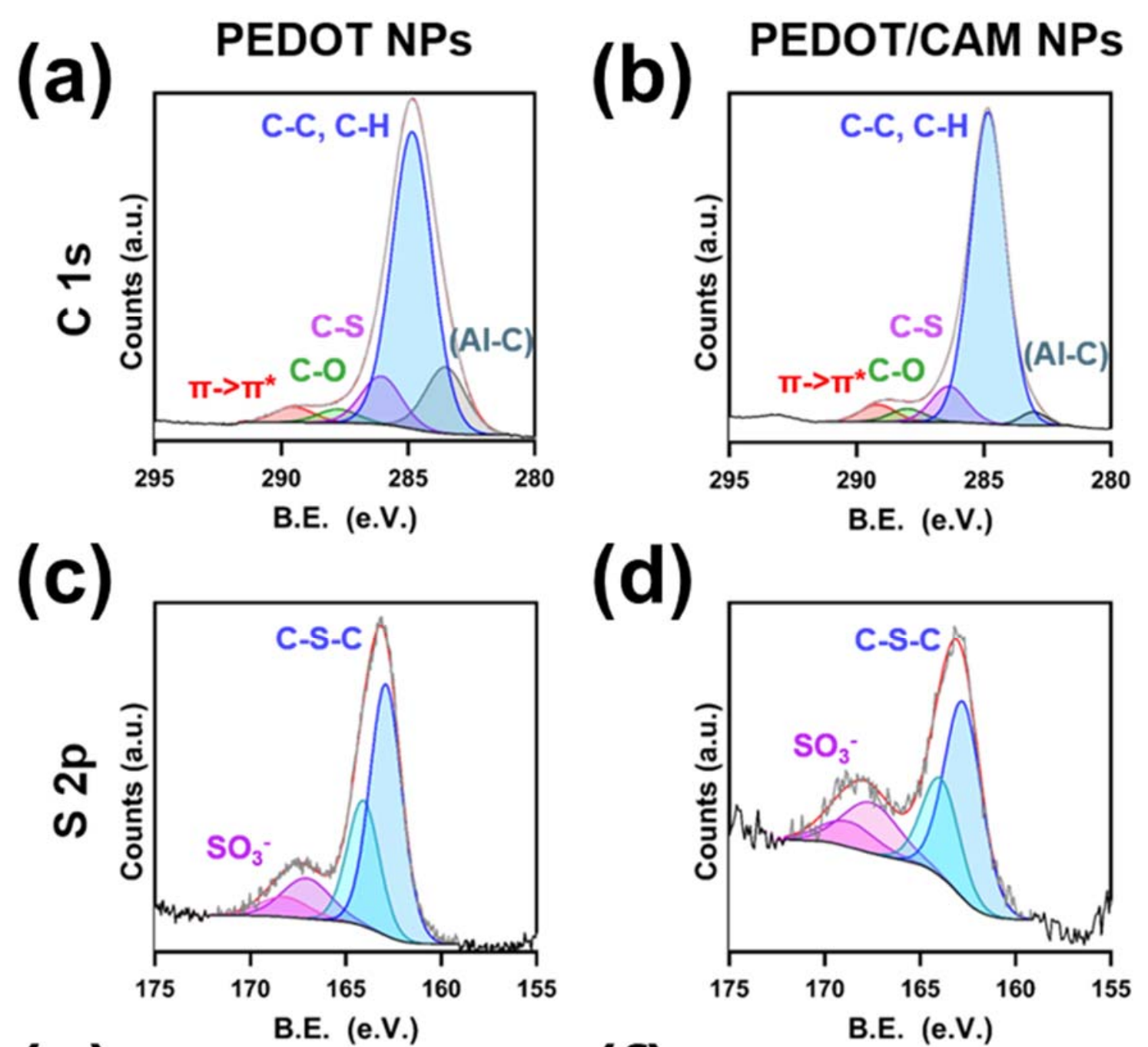

(d)

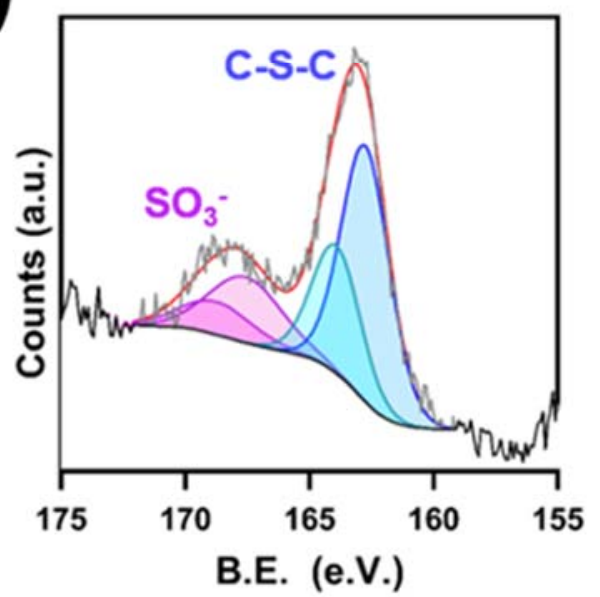

(e)

(f)

B.E. (e.V.)
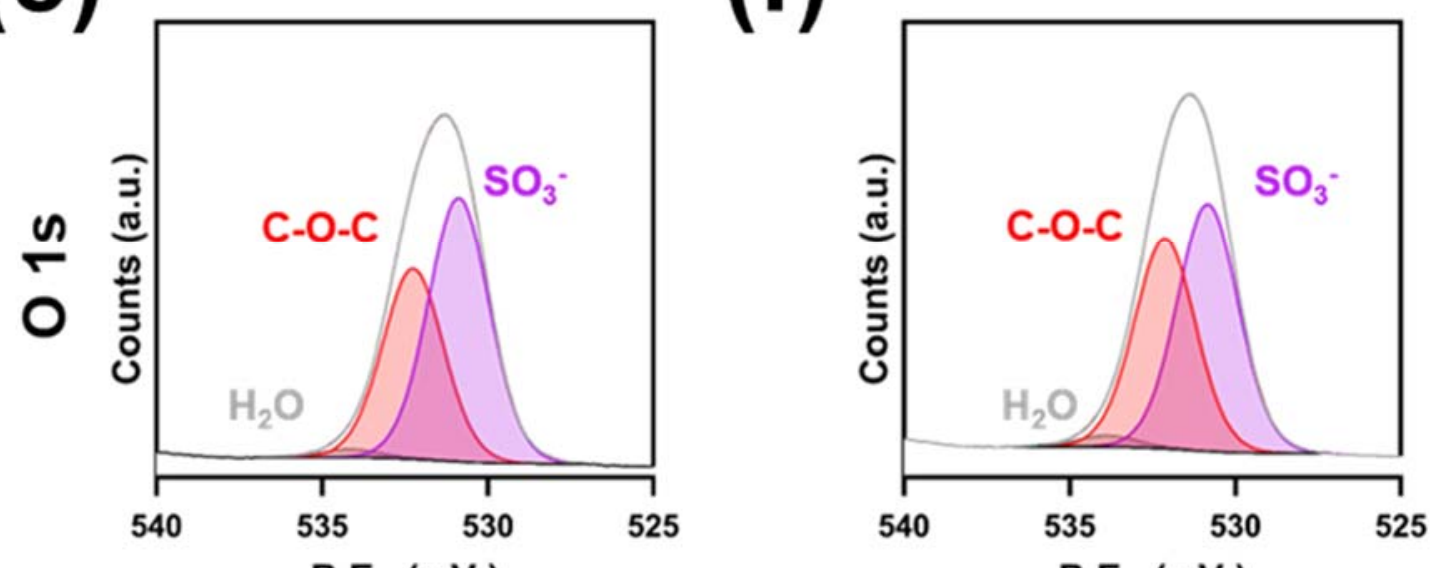

B.E. (e.V.)

B.E. (e.V.)

Figure S5. High-resolution XPS spectra of $(a, b) C$ 1s, (c, d) S 2p and (e, f) O 1s regions for (a, c, e) PEDOT and (b, d, f) PEDOT/CAM NPs. 


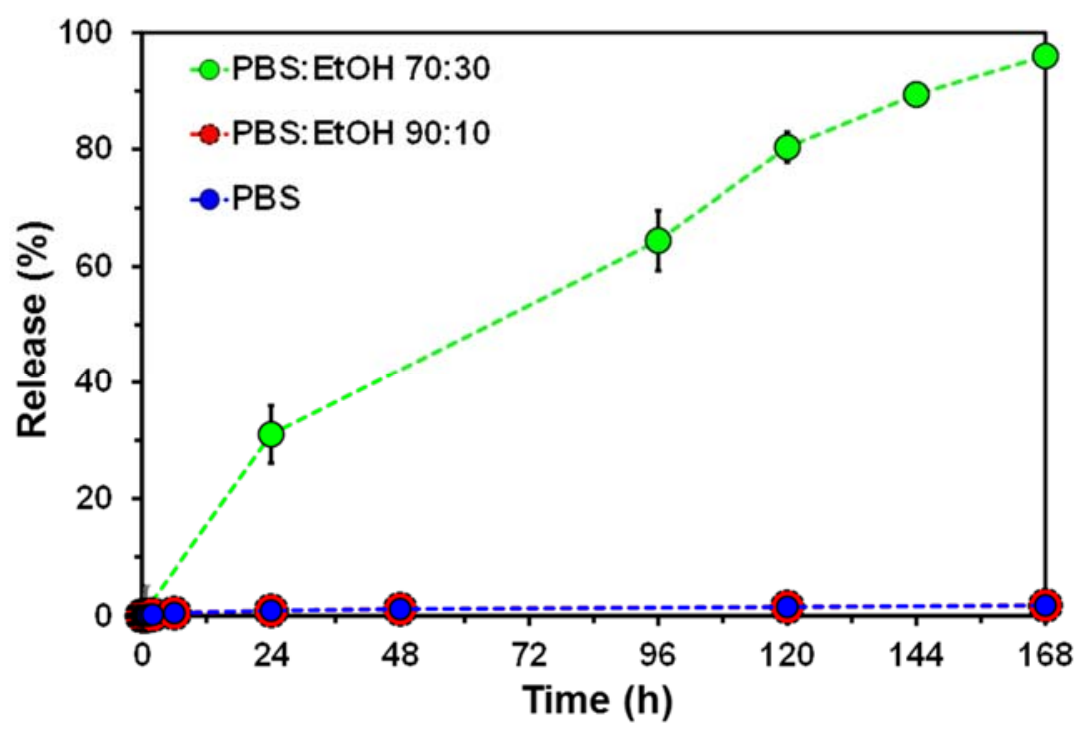

Figure S6. Drug release profiles from PEDOT/CAM NPs in PBS, PBS:EtOH 90:10 and PBS:EtOH 30:70 at $37^{\circ} \mathrm{C}$. The profiles shown in this graphic have been extracted from those displayed in Figure 4 by imposing a common starting point: release of $0 \%$ at the starting period $(\mathrm{t}=0 \mathrm{~h})$ in each environment. 
WILEY-VCH

(a)

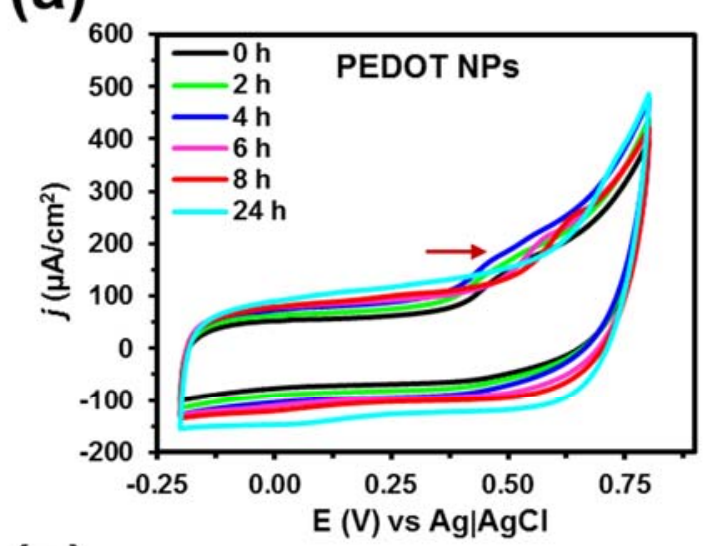

(c)

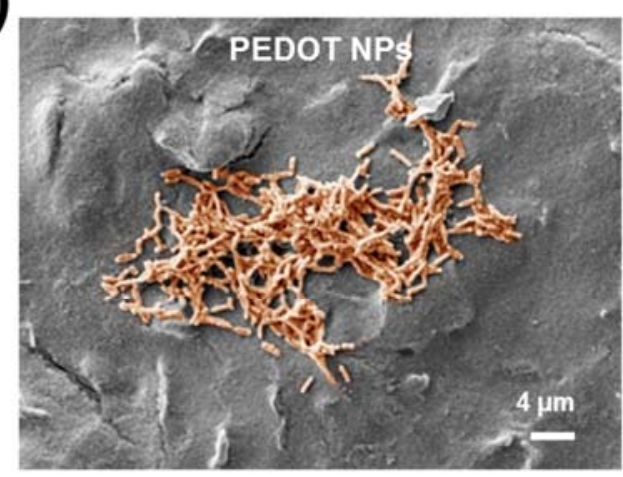

(e)

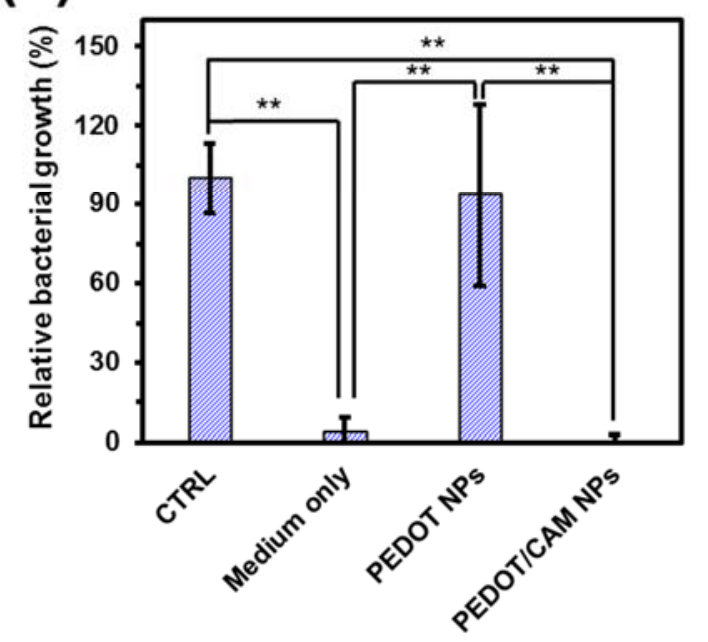

(b)

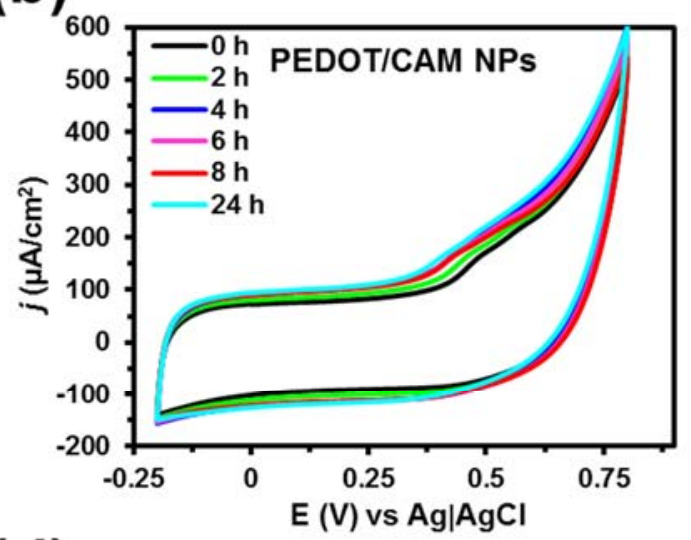

(d)

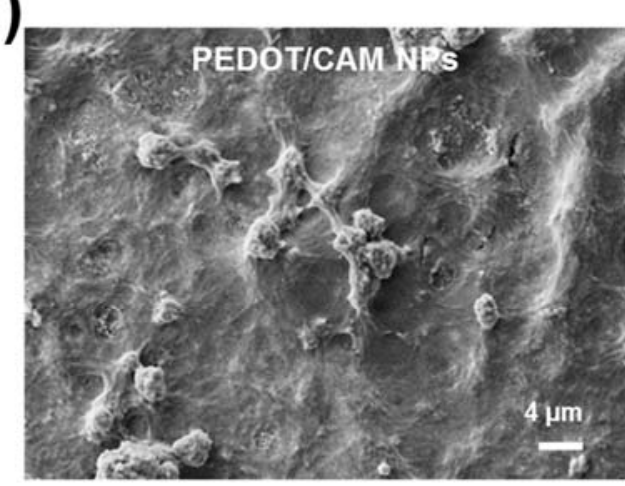

(f)

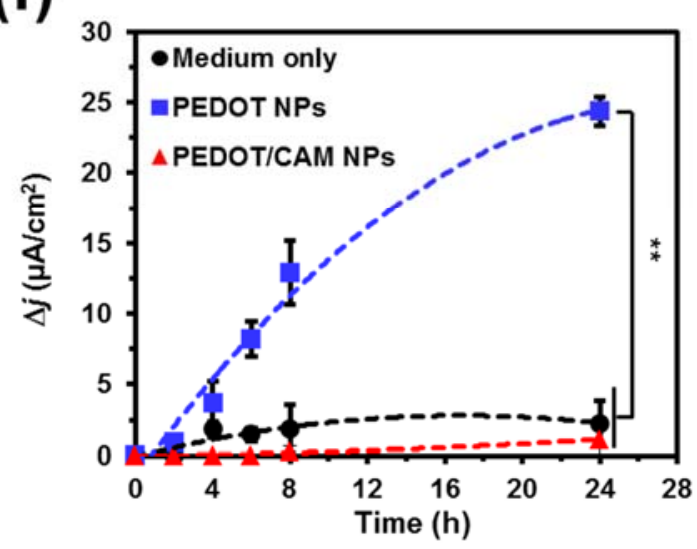

Figure S7. Cyclic voltammograms for SPCEs coated with (a) PEDOT and (b) PEDOT/CAM NPs recorded at different incubation times $(0,2,4,6,8$ and $24 \mathrm{~h})$ in the culture medium ( $\mathrm{NaHCO}_{3}$-supplemented DMEM) with E. coli. Initial and final potentials: $-0.20 \mathrm{~V}$; reversal potential: $0.80 \mathrm{~V}$; and scan rate: $100 \mathrm{mV} / \mathrm{s}$. SEM micrograph of the surface of electrodes coated with (c) PEDOT and (d) PEDOT/CAM NPs after $24 \mathrm{~h}$ in presence of E. coli (artificially coloured in orange). (e) Relative bacterial growth calculated through the variation of the absorbance at $600 \mathrm{~nm}$ after $24 \mathrm{~h}$ of culture. (f) Variation in peak current density with the incubation time relative to $0 \mathrm{~h}$ for the voltammograms displayed in (a) and (b). The statistical comparison of values in (e) and (f) was based on one-way ANOVA with multicomparison test, with $\mathrm{p}<0.0001(* * * *), \mathrm{p}<0.001(* * *)$, and $\mathrm{p}<0.01(* *)$. 


\section{WILEY-VCH}

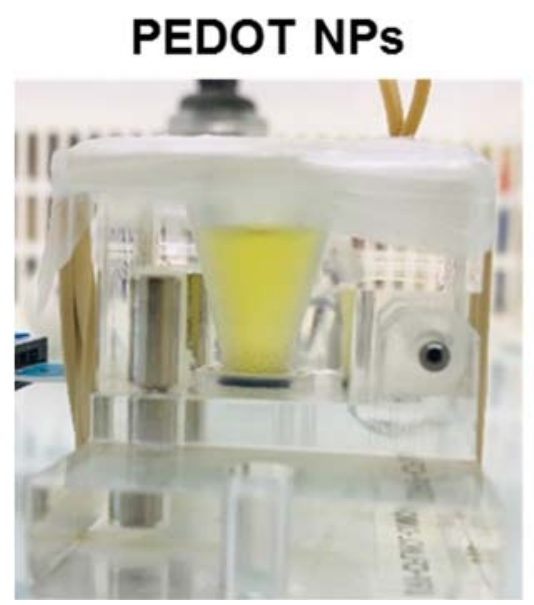

\section{PEDOT/CAM NPS}

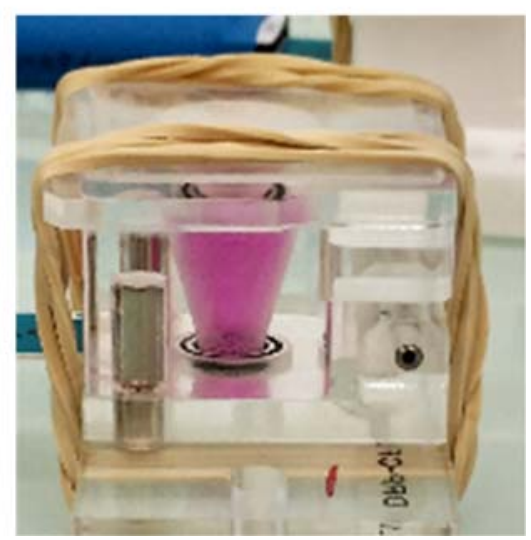

Figure S8. Color of the cell culture medium, which contain phenol red, after $24 \mathrm{~h}$ of bacterial incubation in the presence of PEDOT (left) or PEDOT/CAM NPs (right). The yellowish and pink colors indicate acid and basic pHs, respectively, which are consistent with bacteria growth and inhibition, respectively. 